This is a self-archived version of an original article. This version may differ from the original in pagination and typographic details.

Author(s): Attouchi, Amal; Ruosteenoja, Eero

Title: Remarks on regularity for $p$-Laplacian type equations in non-divergence form

Year: 2018

Version: Accepted version (Final draft)

Copyright: (c) 2018 Elsevier Inc.

Rights: $C C B Y-N C-N D 4.0$

Rights url: https://creativecommons.org/licenses/by-nc-nd/4.0/

Please cite the original version:

Attouchi, A., \& Ruosteenoja, E. (2018). Remarks on regularity for p-Laplacian type equations in non-divergence form. Journal of Differential Equations, 265(5), 1922-1961.

https://doi.org/10.1016/j.jde.2018.04.017 


\title{
REMARKS ON REGULARITY FOR $p$-LAPLACIAN TYPE EQUATIONS IN NON-DIVERGENCE FORM
}

\author{
AMAL ATTOUCHI AND EERO RUOSTEENOJA
}

\begin{abstract}
We study a singular or degenerate equation in non-divergence form modeled by the $p$-Laplacian,

$$
-|D u|^{\gamma}\left(\Delta u+(p-2) \Delta_{\infty}^{N} u\right)=f \quad \text { in } \quad \Omega .
$$

We investigate local $C^{1, \alpha}$ regularity of viscosity solutions in the full range $\gamma>-1$ and $p>1$, and provide local $W^{2,2}$ estimates in the restricted cases where $p$ is close to 2 and $\gamma$ is close to 0 .
\end{abstract}

\section{INTRODUCTION}

In this paper we study local regularity properties of solutions of the following nonlinear PDE,

$$
-|D u|^{\gamma} \Delta_{p}^{N} u:=-|D u|^{\gamma}\left(\Delta u+(p-2) \Delta_{\infty}^{N} u\right)=f \quad \text { in } \Omega,
$$

where $\gamma \in(-1, \infty), p \in(1, \infty), f \in C(\Omega), \Omega \subset \mathbb{R}^{n}$ with $n \geq 2$, and $\Delta_{\infty}^{N} u:=\left\langle D^{2} u \frac{D u}{|D u|}, \frac{D u}{|D u|}\right\rangle$ is the normalized infinity Laplacian. Equation (1.1) is degenerate when $\gamma>0$ and singular when $-1<\gamma \leq 0$, and it is appropriate to use the concept of viscosity solution. The operator $|D u|^{\gamma} \Delta_{p}^{N} u$ is a generalization of the standard $p$-Laplacian $\Delta_{p} u:=\operatorname{div}\left(|D u|^{p-2} D u\right)$ with a more flexible gradient dependence. As special cases we mention the variational $p$-Laplace equation $-\Delta_{p} u=f$ when $\gamma=p-2$, and the nonvariational normalized $p$-Laplace equation $-\Delta_{p}^{N} u=f$ when $\gamma=0$.

Non-variational operators modeled by the $p$-Laplacian have gained increasing interest during the last 15 years. For $C^{2}$ domains with $W^{2, \infty}$ boundary data, Birindelli and Demengel $[7,8,10]$ showed global Hölder and local Lipschitz estimates for a class of fully nonlinear elliptic equations including equation (1.1) by using Ishii-Lions' method. As a consequence of Harnack estimates, Dávila, Felmer and Quaas [23, Theorem 1.2] proved Hölder estimates up to the boundary for the singular case $\gamma \leq 0$ and $p>1$ with Hölder continuous boundary data and domains satisfying a uniform exterior cone condition.

Date: October 23, 2017.

2010 Mathematics Subject Classification. 35J60, 35B65, 35J92.

Key words and phrases. $p$-Laplacian, viscosity solutions, local $C^{1, \alpha}$ regularity, integrability of second derivatives. 
Birindelli and Demengel [9] and Imbert and Silvestre [30] proved $C^{1, \alpha}$ regularity results for related equations. In [9, Proposition 3.6] the authors proved Hölder regularity of the gradient for solutions of (1.1) for $\gamma \leq 0$ and $p \geq 2$ by using approximations and a fixed point argument. In [30] the authors used improvement of flatness to show local $C^{1, \alpha}$ regularity in the degenerate case $\gamma>0$ for viscosity solutions of the equation $|D u|^{\gamma}\left(F\left(x, D^{2} u\right)\right)=f$, where $F$ is a uniformly elliptic operator, and the result was extended to the full range $\gamma>-1$ in [11]. Notice that this result does not cover equation (1.1) because of discontinuous gradient dependence. However, for the special case of radial solutions, Birindelli and Demengel [10] showed $C^{1, \alpha}$ regularity of solutions of (1.1) for $\gamma>-1$ and $p>1$.

In this paper we extend these regularity results to viscosity solutions of (1.1) when $\gamma>-1$ and $p>1$, and provide some results on the existence and the integrability of the second derivatives. Our first result concerns the interior regularity of the gradient.

Theorem 1.1. Let $\gamma>-1, p>1$, and $f \in L^{\infty}(\Omega) \cap C(\Omega)$ be Hölder continuous when $\gamma<0$. Then there exists $\alpha=\alpha(p, n)>0$ such that any viscosity solution $u$ of (1.1) is in $C_{\mathrm{loc}}^{1, \alpha}(\Omega)$, and for any $\Omega^{\prime} \subset \subset \Omega$,

$$
[u]_{C^{1, \alpha}\left(\Omega^{\prime}\right)} \leq C=C\left(p, n, d, \gamma, d^{\prime},\|u\|_{L^{\infty}(\Omega)},\|f\|_{L^{\infty}(\Omega)}\right),
$$

where $d=\operatorname{diam}(\Omega)$ and $d^{\prime}=\operatorname{dist}\left(\Omega^{\prime}, \partial \Omega\right)$.

The structure of the proof is standard. An iterative argument leads us to analyze compactness and regularity of deviations of solutions $u$ from planes, $w(x)=u(x)-q \cdot x$ for some $q \in \mathbb{R}^{n}$, and the key estimate for these deviations is called improvement of flatness, Lemma 3.5. The main difficulty is to obtain Arzelà-Ascoli type compactness for deviations $u(x)-q \cdot x$ by using both Ishii-Lions' method and Alexandrov-Bakelman-Pucci (ABP for short) estimate in the proofs of Lemmas 3.1, 3.2, and 3.3.

Our second result concerns integrability of the second derivatives when $\gamma$ is negative and the range of $p$ is restricted.

Theorem 1.2. Let $\gamma \in(-1,0], p \in\left(1,3+\frac{2}{n-2}\right)$, and $f \in L^{\infty}(\Omega)$ be continuous. Then any viscosity solution $u$ of $(1.1)$ belongs to $W_{\mathrm{loc}}^{2,2}(\Omega)$, and for any $\Omega^{\prime \prime} \subset \subset \Omega^{\prime} \subset \subset \Omega$, we have

$$
\|u\|_{W^{2,2}\left(\Omega^{\prime \prime}\right)} \leq C=C\left(p, n, \gamma, d^{\prime}, d^{\prime \prime},\|u\|_{L^{\infty}(\Omega)},\|f\|_{L^{\infty}(\Omega)}\right),
$$

where $d^{\prime}=\operatorname{diam}\left(\Omega^{\prime}\right)$ and $d^{\prime \prime}=\operatorname{dist}\left(\Omega^{\prime \prime}, \partial \Omega^{\prime}\right)$.

The proof starts from the observation that when $\gamma \leq 0$, viscosity solutions of (1.1) are viscosity solutions of the inhomogeneous normalized $p$-Laplace equation with a drift, $-\Delta_{p}^{N} u=f|D u|^{-\gamma}$, see Lemma 2.4. This equation is singular but uniformly elliptic. The main idea is to regularize the equation (see equation (4.7)) and use the Cordes condition, see Lemma 4.1. This condition guarantees a uniform estimate for solutions of regularized problems, 
which together with equi-Hölder continuity and compactness argument gives a local $W^{2,2}$ estimate for solutions of equation (1.1).

Obtaining integrability for second derivatives in the case $\gamma>0$ is harder because of the degeneracy of equation (1.1). However, for a small $\gamma$ and $p$ close to two, we obtain local $W^{2,2}$ estimate by again using an approximation with uniformly elliptic regularized problems, and considering equation (1.1) as a perturbation of the $\gamma$-Laplacian.

Theorem 1.3. Assume that $f \in W^{1,1}(\Omega) \cap L^{\infty}(\Omega)$ is continuous and $\gamma \in$ $(0, \beta]$, where $\beta \in(0,1)$ and

$$
1-\beta-\sqrt{n}|p-2-\gamma|-\beta(p-2-\gamma)^{+}>0 .
$$

Then any viscosity solution $u$ of (1.1) belongs to $W_{\mathrm{loc}}^{2,2}(\Omega)$, and for any $\Omega^{\prime \prime} \subset \subset \Omega^{\prime} \subset \subset \Omega$, we have

$$
\|u\|_{W^{2,2}\left(\Omega^{\prime \prime}\right)} \leq C\left(p, n, \gamma, \beta, d^{\prime}, d^{\prime \prime},\|u\|_{L^{\infty}(\Omega)},\|f\|_{L^{\infty}(\Omega)},\|f\|_{W^{1,1}(\Omega)}\right),
$$

where $d^{\prime}=\operatorname{diam}\left(\Omega^{\prime}\right)$ and $d^{\prime \prime}=\operatorname{dist}\left(\Omega^{\prime \prime}, \partial \Omega^{\prime}\right)$.

This paper is organized as follows. In Section 2 we define viscosity solutions for the problem (1.1). In Section 3 we prove Theorem 1.1, and in Section 4 Theorems 1.2 and 1.3.

Acknowledgement. A.A. is supported by the Academy of Finland, project number 307870 .

\section{Preliminaries And Definitions of SOlutions}

In this section we define viscosity solutions of equation (1.1) and fix the notation. For $\gamma>0$, the operator $|D u|^{\gamma}\left(\Delta u+(p-2) \Delta_{\infty}^{N} u\right)$ is continuous, and we can use the standard definition of viscosity solutions, see [32]. For $\gamma \leq 0$, the operator (1.1) is undefined when $D u=0$, where it has a bounded discontinuity when $\gamma=0$ and is very singular when $\gamma<0$. This can be remediated adapting the notion of viscosity solution using the upper and lower semicontinuous envelopes (relaxations) of the operator, see [20]. The definition for viscosity solutions for the normalized $p$-Laplacian $(\gamma=0)$ is the following.

Definition 2.1. Let $\Omega$ be a bounded domain and $1<p<\infty$. An upper semicontinuous function $u$ is a viscosity subsolution of the equation $-\Delta_{p}^{N} u=$ $f$ if for all $x_{0} \in \Omega$ and $\phi \in C^{2}(\Omega)$ such that $u-\phi$ attains a local maximum at $x_{0}$, one has

$$
\begin{cases}-\Delta_{p}^{N} \phi\left(x_{0}\right) \leq f\left(x_{0}\right), & \text { if } D \phi\left(x_{0}\right) \neq 0, \\ -\Delta \phi\left(x_{0}\right)-(p-2) \lambda_{\max }\left(D^{2} \phi\left(x_{0}\right)\right) \leq f\left(x_{0}\right), & \text { if } D \phi\left(x_{0}\right)=0 \text { and } p \geq 2, \\ -\Delta \phi\left(x_{0}\right)-(p-2) \lambda_{\min }\left(D^{2} \phi\left(x_{0}\right)\right) \leq f\left(x_{0}\right), & \text { if } D \phi\left(x_{0}\right)=0 \text { and } 1<p<2 .\end{cases}
$$

$A$ lower semicontinuous function $u$ is a viscosity supersolution of (1.1) if for all $x_{0} \in \Omega$ and $\phi \in C^{2}(\Omega)$ such that $u-\phi$ attains a local minimum at 
$x_{0}$, one has

$\begin{cases}-\Delta_{p}^{N} \phi\left(x_{0}\right) \geq f\left(x_{0}\right), & \text { if } D \phi\left(x_{0}\right) \neq 0, \\ -\Delta \phi\left(x_{0}\right)-(p-2) \lambda_{\min }\left(D^{2} \phi\left(x_{0}\right)\right) \geq f\left(x_{0}\right), & \text { if } D \phi\left(x_{0}\right)=0 \text { and } p \geq 2, \\ -\Delta \phi\left(x_{0}\right)-(p-2) \lambda_{\max }\left(D^{2} \phi\left(x_{0}\right)\right) \geq f\left(x_{0}\right), & \text { if } D \phi\left(x_{0}\right)=0 \text { and } 1<p<2 .\end{cases}$

We say that $u$ is a viscosity solution of (1.1) in $\Omega$ if it is both a viscosity sub- and supersolution.

The following definition for the case $\gamma<0$ is adapted from the definition used by Julin and Juutinen in [34] for the singular p-Laplacian.

Definition 2.2. Let $\Omega$ be a bounded domain, $1<p<\infty$, and $-1<\gamma<0$. An upper semicontinuous function $u$ is a viscosity subsolution of (1.1) if $u \not \equiv \infty$ and if for all $\phi \in C^{2}(\Omega)$ such that $u-\phi$ attains a local maximum at $x_{0}$ and $D \phi(x) \neq 0$ for $x \neq x_{0}$, one has

$$
\lim _{r \rightarrow 0} \inf _{\substack{x \in B_{r}\left(x_{0}\right), x \neq x_{0}}}\left(-|D \phi(x)|^{\gamma} \Delta_{p}^{N} \phi(x)\right) \leq f\left(x_{0}\right) .
$$

A lower semicontinuous function $u$ is a viscosity supersolution of (1.1) if $u \not \equiv \infty$ and for all $\phi \in C^{2}(\Omega)$ such that $u-\phi$ attains a local minimum at $x_{0}$ and $D \phi(x) \neq 0$ for $x \neq x_{0}$, one has

$$
\lim _{r \rightarrow 0} \sup _{\substack{x \in B_{r}\left(x_{0}\right) \\ x \neq x_{0}}}\left(-|D \phi(x)|^{\gamma} \Delta_{p}^{N} \phi(x)\right) \geq f\left(x_{0}\right) .
$$

We say that $u$ is a viscosity solution of (1.1) in $\Omega$ if it is both a viscosity sub- and supersolution.

Another definition of solutions was proposed by Birindelli and Demengel in $[6,7,8]$. This definition of solution is an adaptation to the elliptic case of the notion of solution that was introduced by Chen, Giga and Goto [16] and Evans and Spruck [26] for singular problems. This is a variation of the usual notion of viscosity solution for (1.1) that avoids testing with functions having vanishing gradient at the testing point.

Definition 2.3. Let $-1<\gamma<0$ and $p>1$. A lower semicontinuous function $u$ is a viscosity super-solution of (1.1) in $\Omega$, if for every $x_{0} \in \Omega$ one of the following conditions hold.

i) Either for all $\phi \in C^{2}(\Omega)$ such that $u-\phi$ has a local minimum at $x_{0}$ and $D \phi\left(x_{0}\right) \neq 0$, we have

$$
-\left|D \phi\left(x_{0}\right)\right|^{\gamma} \Delta_{p}^{N} \phi\left(x_{0}\right) \geq f\left(x_{0}\right) .
$$

ii) Or there is an open ball $B\left(x_{0}, \delta\right) \subset \Omega, \delta>0$ such that $u$ is constant in $B\left(x_{0}, \delta\right)$ and

$$
0 \geq f(x) \quad \forall x \in B\left(x_{0}, \delta\right) .
$$

An upper semicontinuous function $u$ is a viscosity sub-solution of (1.1) in $\Omega$ if for all $x_{0} \in \Omega$ one of the following conditions hold. 
i) Either for all $\phi \in C^{2}(\Omega)$ such that $u-\phi$ reaches a local maximum at $x_{0}$ and $D \phi\left(x_{0}\right) \neq 0$, we have

$$
\left.-|D \phi(x)|^{\gamma} \Delta_{p}^{N} \phi(x)\right) \leq f\left(x_{0}\right) .
$$

ii) Either there exists an open ball $B\left(x_{0}, \delta\right) \subset \Omega, \delta>0$ such that $u$ is constant in $B\left(x_{0}, \delta\right)$ and

$$
0 \leq f(x) \quad \forall x \in B\left(x_{0}, \delta\right) .
$$

We are going to show that these two definitions are equivalent. We show first that if $u$ is a supersolution of (1.1) in the sense of Definition 2.2, then it is a supersolution in the sense of Definition 2.3. The case $i$ ) is immediate, so we only need to consider the case where $u$ is constant in a ball $B\left(x_{0}, \delta\right)$. We see that for all $y \in B\left(x_{0}, \delta\right)$, the function $\phi(x)=u(y)-|x-y|^{q}$ with $q>\frac{\gamma+2}{\gamma+1}>2$ is a smooth test function with an isolated critical point, and it holds

$$
\lim _{r \rightarrow 0} \sup _{\substack{z \in B_{r}(y) \\ z \neq y}}-|D \phi(z)|^{\gamma} \Delta_{p}^{N} \phi(z)=0
$$

so that $f \leq 0$ in $B\left(x_{0}, \delta\right)$, and the statement follows.

Now we assume that $u$ is a supersolution in the sense of Definition 2.3. Let $x_{0} \in \Omega$ and $\phi$ an admissible test function such that $u-\phi$ has a local minimum at $x_{0}$. We assume without a loss of generality, that $x_{0}$ is a strict local minimum in $B\left(x_{0}, \eta\right)$. If $D \phi\left(x_{0}\right) \neq 0$, then the conclusion is immediate. If $D \phi\left(x_{0}\right)=0$ and $\phi$ has only one critical point in a neighbourhood $B\left(x_{0}, r_{0}\right)$ of $x_{0}$, we have to consider two cases: either $u$ is constant or not. If $u$ is constant in a small ball around $x_{0}$, then $u$ is smooth and it follows that $D^{2} \phi\left(x_{0}\right) \leq 0$. Using that

$$
\begin{array}{ll}
-\Delta_{p}^{N} \phi(x) \geq-\Delta \phi(x)-(p-2) \lambda_{\max }\left(D^{2} \phi(x)\right) & \text { if } p \geq 2, \\
-\Delta_{p}^{N} \phi(x) \geq-\Delta \phi(x)-(p-2) \lambda_{\min }\left(D^{2} \phi(x)\right) & \text { if } p<2,
\end{array}
$$

we get that for $p \geq 2$

$$
\lim _{r \rightarrow 0} \sup _{\substack{x \in B_{r}\left(x_{0}\right), x \neq x_{0}}}-\Delta_{p}^{N} \phi(x) \geq \lim _{r \rightarrow 0} \sup _{\substack{x \in B_{r}\left(x_{0}\right), x \neq x_{0}}}-\Delta \phi(x)-(p-2) \lambda_{\max }\left(D^{2} \phi(x)\right) \geq 0,
$$

and for $1<p<2$

$\lim _{r \rightarrow 0} \sup _{\substack{x \in B_{r}\left(x_{0}\right), x \neq x_{0}}}-\Delta_{p}^{N} \phi(x) \geq \lim _{r \rightarrow 0} \sup _{\substack{x \in B_{r}\left(x_{0}\right), x \neq x_{0}}}-\Delta \phi(x)-(p-2) \lambda_{\min }\left(D^{2} \phi(x)\right) \geq 0$,

where we used that

$$
-\Delta \phi\left(x_{0}\right)-(p-2) \lambda_{\max }\left(D^{2} \phi\left(x_{0}\right)\right) \geq 0
$$

and

$$
-\Delta \phi\left(x_{0}\right)-(p-2) \lambda_{\min }\left(D^{2} \phi\left(x_{0}\right)\right) \geq 0 .
$$


It follows that

$$
\lim _{r \rightarrow 0} \sup _{\substack{x \in B_{r}\left(x_{0}\right) \\ x \neq x_{0}}}-|D \phi(x)|^{\gamma} \Delta_{p}^{N} \phi(x) \geq 0 .
$$

Since we have $f \leq 0$ at $x_{0}$, it follows that

$$
\lim _{r \rightarrow 0} \sup _{\substack{x \in B_{r}\left(x_{0}\right) \\ x \neq x_{0}}}-|D \phi(x)|^{\gamma} \Delta_{p}^{N} \phi(x) \geq f\left(x_{0}\right) .
$$

Next we consider the case where $u$ is not constant in any ball around $x_{0}$. We use the argument of [23, Lemma 2.1]. For $y \in B(0, r)$ where $r>0$ is small enough, we consider the function $\phi_{y}(x)=\phi(x+y)$. Then we have that $u-\phi_{y}$ reaches a local minimum at some point $x_{y}$ in $B\left(x_{0}, \eta\right)$. We can show that there exists a sequence $y_{n} \rightarrow 0$ such that $D \phi_{y_{n}}\left(x_{y_{n}}\right) \neq 0$ for all $n$. Testing the equation at $x_{y_{n}}$ we get that for $n$ large enough

$$
\sup _{\substack{z \in B_{r}\left(x_{0}\right), z \neq x_{0}}}-|D \phi(z)|^{\gamma} \Delta_{p}^{N} \phi(z) \geq-\left|D \phi_{y_{n}}\left(x_{y_{n}}\right)\right|^{\gamma} \Delta_{p}^{N} \phi_{y_{n}}\left(x_{y_{n}}\right) \geq f\left(x_{y_{n}}\right) .
$$

Letting $r \rightarrow 0$ and $n \rightarrow \infty$, we get that

$$
\lim _{r \rightarrow 0} \sup _{\substack{x \in B_{r}\left(x_{0}\right), x \neq x_{0}}}-|D \phi(x)|^{\gamma} \Delta_{p}^{N} \phi(x) \geq f\left(x_{0}\right) .
$$

If such a sequence $\left(y_{n}\right)$ does not exist, then it follows by the property that $x_{0}$ is the only critical point of $\phi$ in a small neighborhood of $x_{0}$, that for all $y \in B(0, r)$, we must have $x_{y}+y=x_{0}$ and also that for all $y \in B(0, r)$ and $x \in B\left(x_{0}, \eta\right)$ we have

$$
u\left(x_{0}-y\right)-\phi\left(x_{0}\right)=u\left(x_{y}\right)-\phi_{y}\left(x_{y}\right) \leq u(x)-\phi_{y}(x)=u(x)-\phi(x+y) .
$$

That is,

$$
u\left(x_{0}-y\right)-u(x) \leq \phi\left(x_{0}\right)-\phi(x+y) .
$$

For a vector $h \in B(0, r)$ and a unit vector $e_{i}$, taking $x=x_{0}+h$ and $y=-h-t e_{i}\left(\right.$ resp. $x=x_{0}+h+t e_{i}$ and $\left.y=-h\right)$ in (2.6), dividing by $t>0$ and taking limsup (resp. liminf), we see that $u$ has directional derivative near $x_{0}$, that vanishes everywhere in the neighborhood. This implies that $u$ is constant around $x_{0}$, which is a contradiction. We can see from the proof that the three definitions are also equivalent when $\gamma=0$. When the operator is continuous $(\gamma>0)$, then the definitions 2.2 and 2.3 are equivalent with the usual definition of viscosity solution (see [23, Lemma 2.1]).

Next we observe that the singular case can be reduced to the study of the normalized $p$-Laplacian problem with a drift.

Lemma 2.4. Let $\gamma \in(-1,0)$. Assume that $u$ is a viscosity solution to (1.1). Then $u$ is a viscosity solution to

$$
-\Delta_{p}^{N} u=f|D u|^{-\gamma} .
$$


Proof. It is sufficient to prove the super-solution property since the subsolution property is similar. We give the proof for $p>2$ the other case being analogous. Let $\phi$ be a test function touching $u$ strictly from below at $x_{0}$. The case $D \phi\left(x_{0}\right) \neq 0$ is immediately clear, so we focus on the case $D \phi\left(x_{0}\right)=0$.

We distinguish two cases: either $D^{2} \phi\left(x_{0}\right)$ is invertible or not. If it is, then $x_{0}$ is the only critical point of $\phi$ in a small ball around $x_{0}$. In this case using the definition of $x_{0}$, we have that for all $\varepsilon>0$ there exist $r_{0}$ such for $r \leq r_{0}$ there exist $x_{r} \rightarrow x_{0}$ such that $D \phi\left(x_{r}\right) \neq 0$ and

$$
-\left|D \phi\left(x_{r}\right)\right|^{\gamma} \Delta_{p}^{N} \phi\left(x_{r}\right) \geq f\left(x_{0}\right)-\varepsilon,
$$

hence

$$
-\Delta_{p}^{N} \phi\left(x_{r}\right) \geq\left(f\left(x_{0}\right)-\varepsilon\right)\left|D \phi\left(x_{r}\right)\right|^{-\gamma} .
$$

It follows that

$$
-\Delta \phi\left(x_{r}\right)-(p-2) \lambda_{\min }\left(D^{2} \phi\left(x_{r}\right)\right) \geq\left(f\left(x_{0}\right)-\varepsilon\right)\left|D \phi\left(x_{r}\right)\right|^{-\gamma} .
$$

Letting $\varepsilon, r \rightarrow 0$, we get

$$
-\Delta \phi\left(x_{0}\right)-(p-2) \lambda_{\min }\left(D^{2} \phi\left(x_{0}\right)\right) \geq 0=f\left(x_{0}\right)\left|D \phi\left(x_{0}\right)\right|^{-\gamma} .
$$

Now we suppose that $D^{2} \phi\left(x_{0}\right)$ is not invertible. Then we take a symmetric matrix $B$, semi-positive definite such that $D^{2} \phi\left(x_{0}\right)-\eta B$ is invertible, for all $\eta>0$. We consider the test function

$$
\phi_{\eta}(x)=\phi(x)-\eta\left(x-x_{0}\right) B\left(x-x_{0}\right) .
$$

Applying the previous argument we have

$$
-\Delta \phi_{\eta}\left(x_{0}\right)-(p-2) \lambda_{\min }\left(D^{2}\left(\phi_{\eta}\left(x_{0}\right)\right) \geq 0=f\left(x_{0}\right)\left|D \phi_{\eta}\left(x_{0}\right)\right|^{-\gamma} .\right.
$$

Passing to the limit $\eta \rightarrow 0$ we get the desired result.

The following lemma will be useful in the next section concerning the proof of the improvement of flatness.

Lemma 2.5. Let $\gamma>-1$. Assume that $v$ is a viscosity solution of

$$
-|D w+q|^{\gamma}\left[\Delta w+(p-2) \frac{\left\langle D^{2} w(D w+q),(D w+q)\right\rangle}{|D w+q|^{2}}\right]=0 .
$$

Then $w$ is a viscosity solution of

$$
-\Delta w-(p-2) \frac{\left\langle D^{2} w(D w+q),(D w+q)\right\rangle}{|D w+q|^{2}}=0 .
$$

Proof. We can reduce the problem to the case $q=0$ since $v=w-q \cdot x$ solves $-|D v|^{\gamma} \Delta_{p}^{N} v=0$. It is sufficient to prove the super-solution property since the sub-solution property is similar. Let $\phi$ be a test function touching $u$ strictly from below at $x_{0}$. We use the fact that for the homogeneous case and for $1<p<\infty$, it is enough to test with functions such that $D \phi\left(x_{0}\right) \neq 0$ (see $[35,36])$ and the statement follows. 


\section{LOCAL HÖLDER REGULARITY OF THE GRADIENT}

In this section we give a proof for Theorem 1.1. We assume that $\gamma \in$ $(-1, \infty), p>1$ and $f \in L^{\infty}(\Omega) \cap C(\Omega)$, and we want to show that there exists $\alpha=\alpha(p, n, \gamma)>0$ such that any viscosity solution $u$ of (1.1) is in $C_{\mathrm{loc}}^{1, \alpha}(\Omega)$, and for any $\Omega^{\prime} \subset \subset \Omega$,

$$
[u]_{C^{1, \alpha}\left(\Omega^{\prime}\right)} \leq C=C\left(p, n, \gamma, d, d^{\prime},\|u\|_{L^{\infty}(\Omega)},\|f\|_{L^{\infty}(\Omega)}\right),
$$

where $d=\operatorname{diam}(\Omega)$ and $d^{\prime}=\operatorname{dist}\left(\Omega^{\prime}, \partial \Omega\right)$.

A standard method to investigate the regularity of solutions is through their approximations by linear functions. The goal is to get good estimates of the error of these approximations using compactness method an scaling properties of the equation. We will use the following characterization of $C^{1, \alpha}$ functions: there exists a positive constant $C$ such that for any $x \in \Omega$ and $r>0$, there exists a vector $l$ for which

$$
\underset{y \in B_{r}(x)}{\operatorname{Osc}}(u(y)-u(x)-l \cdot(x-y)) \leq C r^{1+\alpha} .
$$

By rescaling we may assume osc $u \leq 1$, and by proceeding by iteration it is enough to find $\rho \in(0,1)$ such that inequality (3.1) holds true for $r=r_{k}=\rho^{k}$, $l=l_{k}$ and $C=1$. The balls $B_{r}(x)$ for $x \in \Omega$ and $r<\operatorname{dist}(x, \partial \Omega)$ covering the domain $\Omega$, we may work on balls. Moreover, by using a translation argument and the following scaling,

$$
u_{r}(y)=r^{-\frac{\gamma+2}{\gamma+1}} u(x+r y),
$$

we may work on the unit ball $B_{1}(0)$ and prove the regularity only at the origin. Considering $u-u(0)$ if necessary, we may suppose that $u(0)=0$. We also reduce the problem by rescaling. Let

$$
\kappa=\left(2\|u\|_{L^{\infty}\left(B_{1}\right)}+\left(\varepsilon_{0}^{-1}\|f\|_{L^{\infty}\left(B_{1}\right)}\right)^{1 /(1+\gamma)}\right)^{-1} .
$$

Setting $\tilde{u}=\kappa u$, then $\tilde{u}$ satisfies

$$
-|D \tilde{u}|^{\gamma} \Delta_{p}^{N}(\tilde{u})=\tilde{f}
$$

with

$$
\|\tilde{u}\|_{L^{\infty}\left(B_{1}\right)} \leq \frac{1}{2} \quad \text { and } \quad\|\tilde{f}\|_{L^{\infty}\left(B_{1}\right)} \leq \varepsilon_{0} .
$$

Hence, without loss of generality we may assume that $\|u\|_{L^{\infty}\left(B_{1}\right)} \leq 1 / 2$ and $\|f\|_{L^{\infty}\left(B_{1}\right)} \leq \varepsilon_{0}$, where $\varepsilon_{0}=\varepsilon_{0}(p, n, \gamma)$ is chosen later. To prove the inductive step, the strategy is to study the deviations of $u$ from planes, $w(x)=u(x)-q \cdot x$, which satisfy in $B_{1}$

$$
-|D w+q|^{\gamma}\left[\Delta w+(p-2)\left\langle D^{2} w \frac{D w+q}{|D w+q|}, \frac{D w+q}{|D w+q|}\right\rangle\right]=f
$$

in the viscosity sense. The existence of the vector $q_{k+1}$ can be reduced to prove an "improvement of flatness" for solutions of (3.2). The proof of the improvement of flatness consists of two steps. First we show equicontinuity for uniformly bounded solutions of (3.2) in Lemma 3.2 and Lemma 3.3. 
Next, by the Arzelà-Ascoli theorem we get compactness, which, together with the regularity of the limiting solutions (Lemma 3.4), allow to show improvement of flatness for solutions of (3.2) in Lemma 3.5 via a contradiction argument. Finally, we prove $C^{1, \alpha}$ regularity for solutions of (1.1) in Lemma 3.6 by using Lemma 3.5 and iteration.

3.1. Equicontinuity for deviations from planes. First, we need to prove some compactness result for the deviations from planes. We will provide Hölder regularity results independently of $q$ using different arguments for large and small slopes. For large slopes, the strategy is to consider $\tilde{w}=\frac{w}{|q|}$ and to prove that

$$
\|\tilde{w}\|_{C^{\alpha}\left(B_{r}\right)} \leq C(p, n)\left(\|\tilde{w}\|_{L^{\infty}\left(B_{1}\right)}+\frac{\|f\|_{L^{\infty}\left(B_{1}\right)}^{\frac{1}{1+\gamma}}}{|q|}\right) .
$$

We would then immediately deduce that for $|q|>1$

$$
\|w\|_{C^{\alpha}\left(B_{r}\right)} \leq C(p, n)\left(\|w\|_{L^{\infty}\left(B_{1}\right)}+\|f\|_{L^{\infty}\left(B_{1}\right)}^{\frac{1}{1+\gamma}}\right) .
$$

For this purpose, we will need the following technical lemma concerning Hölder regularity of the functions $\tilde{w}$ which are solutions in $B_{1}$ of the following equation :

$$
-|D \tilde{w}+e|^{\gamma}\left[\Delta \tilde{w}+(p-2)\left\langle D^{2} \tilde{w} \frac{D \tilde{w}+e}{|D \tilde{w}+e|}, \frac{D \tilde{w}+e}{|D \tilde{w}+e|}\right\rangle\right]=f|q|^{-\gamma-1},
$$

where $e:=\frac{q}{|q|}$.

Lemma 3.1. Let $\gamma \in(-1, \infty)$ and $p \in(1, \infty)$. Assume that $|q| \neq 0$ and let $\tilde{w}$ be a viscosity solution of $(3.3)$ in $B_{1}$. For all $r \in\left(0, \frac{3}{4}\right)$ and $\beta \in(0,1)$, there exists a constant $C=C(p, n, \gamma, \beta)>0$ such that for all $x, y \in B_{r}$,

$$
|\tilde{w}(x)-\tilde{w}(y)| \leq C\left(\|\tilde{w}\|_{L^{\infty}\left(B_{1}\right)}+\frac{\|f\|_{L^{\infty}\left(B_{1}\right)}^{\frac{1}{1+\gamma}}}{|q|}\right)|x-y|^{\beta} .
$$

For the proof, see Appendix A.

As a consequence of Lemma 3.1, we have

Lemma 3.2. Let $\gamma \in(-1, \infty)$ and $p \in(1, \infty)$. Assume that $|q| \geq 1$ and let $w$ be a viscosity solution to equation (3.2). For all $r \in(0,1)$ and $\beta \in(0,1)$, there exists a constant $C=C(p, n, \gamma, \beta)>0$ such that for all $x, y \in B_{r}$,

$$
|w(x)-w(y)| \leq C\left(\|w\|_{L^{\infty}\left(B_{1}\right)}+\|f\|_{L^{\infty}\left(B_{1}\right)}^{\frac{1}{1+\gamma}}\right)|x-y|^{\beta} .
$$

In the next lemma we use the following notation for Pucci operators:

$$
\mathcal{M}^{+}(X):=\sup _{A \in \mathcal{A}_{\lambda, \Lambda}}-\operatorname{tr}(A X)
$$


and

$$
\mathcal{M}^{-}(X):=\inf _{A \in \mathcal{A}_{\lambda, \Lambda}}-\operatorname{tr}(A X),
$$

where $\mathcal{A}_{\lambda, \Lambda} \subset S^{n}$ is a set of symmetric $n \times n$ matrices whose eigenvalues belong to $[\lambda, \Lambda]$.

The following lemma provides uniform Hölder estimate for small slopes.

Lemma 3.3. For all $r \in(0,1)$, there exist a constant $\beta=\beta(p, n) \in(0,1)$ and a positive constant $C=C\left(p, n, r, \underset{B_{1}}{\operatorname{osc}}(w),\|f\|_{L^{n}\left(B_{1}\right)}\right)$ such that any viscosity solution $w$ of (3.2) with $|q| \leq 1$ satisfies

$$
[w]_{C^{0, \beta}\left(B_{r}\right)} \leq C \text {. }
$$

Proof. Assume first that $\gamma \geq 0$. If $|D w| \geq 2$ then $|D w+q| \geq|| D w|-| q|| \geq 1$ and $|D w+q|^{-\gamma} \leq 1$. Hence we have

$$
\left\{\begin{array}{l}
\mathcal{M}^{+}\left(D^{2} w\right)+|f| \geq 0 \\
\mathcal{M}^{-}\left(D^{2} w\right)-|f| \leq 0
\end{array}\right.
$$

Using the result of [31] (see also [24]), there exists $\beta=\beta(p, n) \in(0,1)$ such that

$$
[w]_{C^{0, \beta}\left(B_{r}\right)} \leq C=C\left(\begin{array}{c}
p, n, r, \underset{B_{1}}{\operatorname{osc}(w)},\|f\|_{L^{n}\left(B_{1}\right)} \\
B_{1}
\end{array} .\right.
$$

Now if $\gamma<0$, then $|D w+q|^{-\gamma} f \leq|D w|+|f|+C(\gamma)|f|^{\frac{1}{1+\gamma}}$, and hence

$$
\left\{\begin{array}{l}
\mathcal{M}^{+}\left(D^{2} w\right)+|D w|+C(\gamma)|f|^{\frac{1}{1+\gamma}}+|f| \geq 0 \\
\mathcal{M}^{-}\left(D^{2} w\right)-|D w|-C(\gamma)|f|^{\frac{1}{1+\gamma}}-|f| \leq 0 .
\end{array}\right.
$$

It follows from the ABP estimate and the Harnack inequality, that $w$ is Hölder continuous and that

$$
[w]_{C^{0, \beta}\left(B_{r}\right)} \leq C=C\left(p, n, \gamma, r, \underset{B_{1}}{\operatorname{osc}(w)},\|f\|_{L^{n}\left(B_{1}\right)}\right) .
$$

3.2. Proof of the improvement of flatness. The next lemma gives uniform Hölder estimates for the limit equation independent of $|a|$, and is needed in the contradiction argument in the proof of the key Lemma 3.5, where we show improvement of flatness. We refer the reader to $[2$, Lemma 3.2 .

Lemma 3.4. Let $v$ be a viscosity solution of

$$
-\Delta v-(p-2)\left\langle D^{2} v \frac{D v+q}{|D v+q|}, \frac{D v+q}{|D v+q|}\right\rangle=0 \quad \text { in } \quad B_{1}
$$

with osc $v \leq 1$. For all $r \in\left(0, \frac{1}{2}\right]$, there exist constants $C_{0}=C_{0}(p, n)>0$ and $\beta_{1}=\beta_{1}(p, n)>0$ such that

$$
[v]_{C^{1, \beta_{1}\left(B_{r}\right)}} \leq C_{0}
$$


Lemma 3.5. Let $\gamma \in(-1, \infty)$ and $p \in(1, \infty)$ and suppose that $f \in C^{\bar{\beta}}\left(B_{1}\right)$ is Hölder continuous when $\gamma<0$. There exist $\varepsilon_{0} \in(0,1)$ and $\rho=\rho(p, n) \in$ $(0,1)$ such that, for any $q \in \mathbb{R}^{n}$ and any viscosity solution $w$ of $(3.2)$ with $\operatorname{osc}_{B_{1}}(w) \leq 1$ and $\|f\|_{L^{\infty}\left(B_{1}\right)} \leq \varepsilon_{0}$, there exists $q^{\prime} \in \mathbb{R}^{n}$ such that

$$
\underset{x \in B_{\rho}}{\operatorname{Osc}}\left(w(x)-q^{\prime} \cdot x\right) \leq \frac{1}{2} \rho .
$$

Proof. An argument by contradiction. Assume that there exist a sequence of functions $\left(f_{j}\right)$ with $\left\|f_{j}\right\|_{L^{\infty}\left(B_{1}\right)} \rightarrow 0$, a sequence of vectors $\left(q_{j}\right)$ and a sequence of viscosity solutions $\left(w_{j}\right)$ with $\operatorname{osc}_{B_{1}}\left(w_{j}\right) \leq 1$ of

$$
-\left|D w_{j}+q_{j}\right|^{\gamma}\left[\Delta w_{j}+(p-2)\left\langle D^{2} w_{j} \frac{D w_{j}+q_{j}}{\left|D w_{j}+q_{j}\right|}, \frac{D w_{j}+q_{j}}{\left|D w_{j}+q_{j}\right|}\right\rangle\right]=f_{j},
$$

such that, for all $q^{\prime} \in \mathbb{R}^{n}$ and any $\rho \in(0,1)$

$$
\underset{x \in B_{\rho}}{\operatorname{osc}}\left(w_{j}(x)-q^{\prime} \cdot x\right)>\frac{\rho}{2} .
$$

Relying on the compactness result of Lemma 3.2 and Lemma 3.3, there exists a continuous function $w_{\infty}$ such that $w_{j} \rightarrow w_{\infty}$ uniformly in $B_{\rho}$ for any $\rho \in(0,1)$. Passing to the limit in $(3.10)$, we have that for any vector $q^{\prime}$,

$$
\underset{x \in B_{\rho}}{\operatorname{Osc}}\left(w_{\infty}(x)-q^{\prime} \cdot x\right)>\frac{\rho}{2} .
$$

We treat separately the cases where the sequence $\left(q_{j}\right)$ is bounded or unbounded. Suppose first that the sequence $\left(q_{j}\right)$ is bounded. Using a compactness argument and relaxed limits, we extract a subsequence $\left(w_{j}\right)$ converging to a limit $w_{\infty}$, which satisfies in $B_{1}$

$-\left|D w_{\infty}+q_{\infty}\right|^{\gamma} \operatorname{tr}\left(\left(I+(p-2) \frac{D w_{\infty}+q_{\infty}}{\left|D w_{\infty}+q_{\infty}\right|} \otimes \frac{D w_{\infty}+q_{\infty}}{\left|D w_{\infty}+q_{\infty}\right|}\right) D^{2} w_{\infty}\right)=0$

in a viscosity sense. (Here $q_{j} \rightarrow q_{\infty}$ up to the same subsequence.) Using the result of Lemma 2.5, we know that viscosity solutions to (3.12) are viscosity solutions to (3.7) and using the regularity result of Lemma 3.4, there exist $\beta_{1}=\beta_{1}(p, n)>0$ and $C_{0}=C_{0}(p, n)>0$ such that

$$
\left\|w_{\infty}\right\|_{C^{1, \beta_{1}\left(B_{1 / 2}\right)}} \leq C_{0}
$$

If the sequence $\left(q_{j}\right)$ is unbounded, we can extract a converging subsequence from $e_{j}=\frac{q_{j}}{\left|q_{j}\right|}$ such that $e_{j} \rightarrow e_{\infty}$.

If $\gamma \geq 0$, we obtain

$$
-\Delta w_{\infty}-(p-2)\left\langle D^{2} w_{\infty} e_{\infty}, e_{\infty}\right\rangle=0 \quad \text { in } \quad B_{1},
$$

with $\left|e_{\infty}\right|=1$. Noticing that equation (3.13) can be written as

$$
-\operatorname{tr}\left(\left(I+(p-2) e_{\infty} \otimes e_{\infty}\right) D^{2} w_{\infty}\right)=0,
$$

we see that equation (3.13) is uniformly elliptic,continuous and linear. Using the regularity result of [13, Corollary 5.7], there is $\beta_{2}=\beta_{2}(p, n)>0$ so that 
$w_{\infty} \in C_{\mathrm{loc}}^{1, \beta_{2}}$ and there exists $C_{0}=C_{0}(p, n)>0$ such that $\left\|w_{\infty}\right\|_{C^{1, \beta_{2}\left(B_{1 / 2}\right)}} \leq$ $C_{0}$.

If $\gamma<0$, we show using a contradiction argument that $\|\left. f_{j}(x)\right|_{C^{\bar{\beta}}\left(B_{1}\right)}\left|q_{j}\right|^{-\gamma}$ is uniformly bounded. Then, by Arzelà-Ascoli's theorem, up to a subsequence, $w_{j} \rightarrow w_{\infty}$ and $f_{j}(x)\left|q_{j}\right|^{-\gamma} \rightarrow h_{\infty}$ locally uniformly. Passing to the limit $j \rightarrow \infty$ in

$$
\begin{aligned}
-\Delta w_{j}-(p-2) & \left\langle D^{2} w_{j} \frac{\left|q_{j}\right|^{-1} D w_{j}+e_{j}}{\left.|| q_{j}\right|^{-1} D w_{j}+e_{j} \mid}, \frac{\left|q_{j}\right|^{-1} D w_{j}+e_{j}}{\left.|| q_{j}\right|^{-1} D w_{j}+e_{j} \mid}\right\rangle \\
& =\left.f_{j}\left|q_{j}\right|^{-\gamma}|| q_{j}\right|^{-1} D w_{j}+\left.e_{j}\right|^{-\gamma},
\end{aligned}
$$

we see that $w_{\infty}$ solves

$$
-\operatorname{tr}\left(\left(I+(p-2) e_{\infty} \otimes e_{\infty}\right) D^{2} w_{\infty}\right)=h_{\infty},
$$

and we conclude that $w_{\infty}$ is in $C^{1, \beta_{2}}$ with a norm $C_{0}$ independent of the slope since $\left\|f_{j}(x)\right\|_{L^{\infty}}\left|q_{j}\right|^{-\gamma}$ is bounded.

Now suppose that $\left\|f_{j}(x)\left|q_{j}\right|^{-\gamma}\right\|_{C^{\bar{\beta}\left(B_{1}\right)}}$ is not bounded. Denoting

$$
b_{j}:=\left.|| f_{j}(x)\right|_{L^{\infty}\left(B_{1}\right)}\left|q_{j}\right|^{-\gamma}
$$

and considering $a_{j}(x):=\frac{w_{j}(x)}{b_{j}}$ we have that $a_{j}$ solves

$$
\begin{aligned}
-\Delta a_{j}-(p-2) & \left\langle D^{2} a_{j} \frac{b_{j}\left|q_{j}\right|^{-1} D a_{j}+e_{j}}{\left.\left|b_{j}\right| q_{j}\right|^{-1} D a_{j}+e_{j} \mid}, \frac{b_{j}\left|q_{j}\right|^{-1} D a_{j}+e_{j}}{\left.\left|b_{j}\right| q_{j}\right|^{-1} D a_{j}+e_{j} \mid}\right\rangle \\
& =\left.f_{j}\left|q_{j}\right|^{-\gamma} b_{j}^{-1}\left|b_{j}\right| q_{j}\right|^{-1} D a_{j}+\left.e_{j}\right|^{-\gamma} .
\end{aligned}
$$

Since $-1-\gamma<0$, we have $\left|q_{j}\right|^{-1} b_{j} \rightarrow 0$. We have that $a_{j}$ converges uniformly to zero and, by passing to the limit in the equation, we get

$$
\lim _{j \rightarrow \infty} f_{j}(x)\left|q_{j}\right|^{-\gamma} b_{j}^{-1}=0,
$$

which is a contradiction since $\left\|f_{j}\left|q_{j}\right|^{-\gamma} b_{j}^{-1}\right\|_{L^{\infty}}=1$ and up to a subsequence the convergence is uniform.

It follows that $w_{\infty} \in C_{\text {loc }}^{1, \beta}$ for $\beta=\min \left(\beta_{1}, \beta_{2}\right)>0$. Choose $\rho \in(0,1 / 2)$ such that

$$
C_{0} \rho^{\beta} \leq \frac{1}{4}
$$

By $C_{\text {loc }}^{1, \beta}$ regularity, there exists a vector $k_{\rho}$ such that

$$
\underset{x \in B_{\rho}}{\operatorname{osc}}\left(w_{\infty}(x)-k_{\rho} \cdot x\right) \leq C_{0} \rho^{1+\beta} \leq \frac{1}{4} \rho .
$$

This contradicts (3.11) so the proof is complete.

Once we have proved the improvement of flatness for deviations from planes, the proof of Theorem 1.1 proceeds by standard iteration. 
Lemma 3.6. Let $\rho$ and $\varepsilon_{0} \in(0,1)$ be as in Lemma 3.5 and let $u$ be a viscosity solution of (1.1) with $\gamma>-1, p>1, \operatorname{Osc}_{B_{1}}(u) \leq 1$ and $\|f\|_{L^{\infty}\left(B_{1}\right)} \leq \varepsilon_{0}$. Then, there exists $\alpha \in\left(0, \frac{1}{1+\gamma}\right]$ such that for all $k \in \mathbb{N}$, there exists $q_{k} \in \mathbb{R}^{n}$ such that

$$
\underset{y \in B_{r_{k}}}{\operatorname{Osc}}\left(u(y)-q_{k} \cdot y\right) \leq r_{k}^{1+\alpha},
$$

where $r_{k}:=\rho^{k}$.

Proof. For $k=0$, the estimate (3.16) follows from the assumption $\operatorname{osc}_{B_{1}}(u) \leq$ 1. Next we take $\alpha \in\left(0, \frac{1}{\gamma+1}\right)$ such that $\rho^{\alpha}>1 / 2$. We assume for $k \geq 0$ that we already constructed $q_{k} \in \mathbb{R}^{n}$ such that (3.16) holds true. To prove the inductive step $k \rightarrow k+1$, we rescale the solution considering for $x \in B_{1}$

$$
w_{k}(x)=r_{k}^{-1-\alpha}\left(u\left(r_{k} x\right)-q_{k} \cdot\left(r_{k} x\right)\right) .
$$

By induction assumption, we have $\underset{B_{1}}{\operatorname{osc}}\left(w_{k}\right) \leq 1$, and $w_{k}$ satisfies

$$
-\left|D w_{k}+\xi_{k}\right|^{\gamma}\left[\Delta w_{k}+(p-2)\left\langle D^{2} w_{k} \frac{D w_{k}+\xi_{k}}{\left|D w_{k}+\xi_{k}\right|}, \frac{D w_{k}+\xi_{k}}{\left|D w_{k}+\xi_{k}\right|}\right\rangle\right]=f_{k},
$$

where $\xi_{k}=\left(q_{k} / r_{k}^{\alpha}\right)$ and $f_{k}(x)=r_{k}^{1-(\gamma+1) \alpha} f\left(r_{k} x\right)$ with $\left\|f_{k}\right\|_{L^{\infty}\left(B_{1}\right)} \leq \varepsilon_{0}$ since $\alpha<\frac{1}{\gamma+1}$. Using the result of Lemma 3.5, there exists $l_{k+1} \in \mathbb{R}^{n}$ such that

$$
\underset{x \in B_{\rho}}{\operatorname{Osc}}\left(w_{k}(x)-l_{k+1} \cdot x\right) \leq \frac{1}{2} \rho .
$$

Setting $q_{k+1}=q_{k}+l_{k+1} r_{k}^{\alpha}$, we get

$$
\underset{x \in B_{r_{k+1}}}{\operatorname{OSC}}\left(u(x)-q_{k+1} \cdot x\right) \leq \frac{\rho}{2} r_{k}^{1+\alpha} \leq r_{k+1}^{1+\alpha} .
$$

Since the estimate (3.16) holds for every $k$, the proof of Theorem 1.1 is complete.

Remark 3.7. 1) For $\gamma \leq 0$, when the boundary data $g \in C^{1, \beta}$ and the domain $\Omega$ has a $C^{1, \beta}$ boundary, the boundary Hölder regularity of the gradient is a direct consequence of the uniform ellipticity of the operator and the result of [44].

2) For $\gamma>0$, the regularity of the gradient up to the boundary could be obtained by adapting the arguments of [11].

3.3. An alternative proof for the regularity of the gradient when $\gamma \leq p-2$. In this range we can rely on known results of weak theory. When $\gamma \leq p-2$, we have that viscosity solutions of (1.1) are viscosity solution of

$$
-\Delta_{p} u=f|D u|^{p-2-\gamma} \text {. }
$$

Since $0 \leq p-2-\gamma<p-1$ and $f$ is bounded, using the result of [48, Theorem 1.4] which generalize the result of Julin and Juutinen [34], we get 
that viscosity solutions to (3.17) are also weak solutions to (3.17). The result of [54] implies that $u \in C_{l o c}^{1, \alpha}(\Omega)$ and

$$
[u]_{C^{1, \alpha}\left(\Omega^{\prime}\right)} \leq C=C\left(p, n, d, \gamma, d^{\prime},\|u\|_{L^{\infty}(\Omega)},\|f\|_{L^{\infty}(\Omega)}\right) .
$$

Hence, if $\gamma$ is negative but $\gamma \leq p-2$, the Hölder continuity of $f$ is not needed. Moreover for a bounded domain $\Omega$ with a $C^{1, \beta}$ boundary, when we complement the equation (1.1) with a boundary condition $g \in C^{1, \beta}(\bar{\Omega})$, then it follows from the result of [41, Theorem 1], that the viscosity solution is in $C^{1, \alpha}(\bar{\Omega})$, with a norm depending only on $n, p, \Omega,\|f\|_{L^{\infty}(\Omega)},\|g\|_{C^{1, \beta}(\bar{\Omega})}$.

Remark 3.8. The assumption that $f$ is Hölder continuous when $p-2<\gamma<$ 0 is probably just a technicality, however we couldn't remove this assumption in order to prove Hölder continuity of the gradient.

Remark 3.9. In the case $0<\gamma \leq p-2$, it is possible to relax the dependance on $f$ in the local $C^{1, \alpha}$ estimate from $L^{\infty}$-norm to $L^{q}$-norm for some $q<\infty$. Here we just outline the idea and refer to [2, Section 4], where a similar technique was used by relying on the paper of Duzaar and Mingione [25].

Fix a viscosity solution $u$ of (1.1) and a small $\lambda>0$, then consider the approximation process which consists in studying the equation

$\begin{cases}-\operatorname{div}\left(\left(\left|D v_{\varepsilon}\right|^{2}+\varepsilon^{2}\right)^{\frac{p-2}{2}} D v_{\varepsilon}\right)=\left(f_{\varepsilon}+\lambda u-\lambda v_{\varepsilon}\right)\left(\left|D v_{\varepsilon}\right|^{2}+\varepsilon^{2}\right)^{\frac{p-2-\gamma}{2}} & \text { in } \Omega \\ v_{\varepsilon}=u & \text { in } \partial \Omega .\end{cases}$ One can provide uniform Lipschitz estimates for $v_{\varepsilon}$ depending on the the $L^{q}$ norm of $f$ for some $q=\max \left(n, \frac{p}{\gamma+2},\right)$ and Lorentz $L(n, 1)$ norm of $f$. Indeed using the fact that $u$ is a weak solution we can control the $L^{p}$ norm of the gradient by a Caccioppoli inequality, and the fact that the $L^{\infty}$ norm of $u$ is controlled by the $L^{n}$ norm of $f$, see the ABP estimate [23, Theorem 1.1] for viscosity solutions of (1.1). Combining these estimates with the computations of [25], we get the uniform estimates which depends on a lower norm of $f$. Then one can prove that

$$
[u]_{C^{1, \alpha}\left(\Omega^{\prime \prime}\right)} \leq C=C\left(p, q, n, d,, \gamma, d^{\prime \prime},\|u\|_{L^{\infty}(\Omega)},\|f\|_{L^{q}(\Omega)}\right),
$$

where $d=\operatorname{diam}(\Omega)$ and $d^{\prime \prime}=\operatorname{dist}\left(\Omega^{\prime \prime}, \partial \Omega^{\prime}\right)$. Here $\Omega^{\prime \prime} \subset \subset \Omega^{\prime} \subset \subset \Omega$.

\section{4. $W^{2,2}$ REGULARITY}

In this section we study the integrability properties of the second derivatives of the viscosity solutions to (1.1). The idea is to divide the study into different cases. When $\gamma \leq 0$, we reduce the problem to the case of the normalized $p$-Laplacian with a continuous right hand term. We rely on the uniform ellipticity of the operator and on the Cordes condition (see Lemma 4.1). When $\gamma>0$, the operator is degenerate and we have to use another trick. Then we study a regularized problem. When $1>\gamma>0$ but still $|\gamma-p-2| \leq \delta$ for some $\delta$ small enough and $p$ close to 2 , we prove uniform 
estimates on the second derivatives of the approximate solutions. This will provide the desired result by passing to the limit problem.

Let us recall some know results and open problems about the existence and integrability of the second derivatives of solutions for $p$-Laplacian type problems. The $W^{2,2}$-estimates for elliptic equations with measurable coefficients in smooth domains were obtained by Bers and Nirenberg [3] in the two dimensional case in 1954, and by Talenti [53] in any dimensions under the Cordes condition. In [14] Campanato established the $W^{2, q}$-estimate for elliptic equations with measurable coefficients in 2 dimension for $q$ in a neighborhood of 2.

There are also some available results for $p$-harmonic functions $[12,55]$ based on difference quotient, which assert that the nonlinear expression of the gradient

$$
|D u|^{\frac{p-2}{2}} D u \in W_{l o c}^{1,2}(\Omega) .
$$

This property and the local boundedness of the gradient guarantee that for $p \in(1,2)$ the second derivative exists and $u \in W_{l o c}^{2,2}(\Omega)$.

For $p>2$, the derivative $\frac{\partial}{\partial x_{i}}\left(|D u|^{\frac{p-2}{2}} \frac{\partial u}{\partial x_{j}}\right)$ exists but the passage to $D^{2} u$ is difficult at the critical points. The existence of second derivatives of $W^{1, p}$-solutions is not clear due to the degeneracy of the problem. In dimension 2 and for $p \in(1, \infty), p$-harmonic function are in $C_{l o c}^{1, \alpha}(\Omega) \cap W_{l o c}^{3, q}(\Omega)$ where $q$ is any number $1 \leq q<\frac{2}{2-\alpha}$, see [33, Theorem 1]. The nonhomogeneous case

$$
-\Delta_{p} u=g
$$

was also treated with partial results. For $g \in L^{r}(\Omega)$ with $r>\max \left(2, \frac{n}{p}\right)$ and $p \in(1, \infty)$, Lou proved in [43] that weak solutions satisfy $|D u|^{p-1} \in$ $W_{l o c}^{1,2}(\Omega)$. Tolksdorf [54] proved $W_{l o c}^{2,2}$-regularity for $p \in(1,2]$ and $g \in L^{\infty}(\Omega)$, see also $[1,49,52]$. The $W_{l o c}^{2,2}$ regularity for $2<p<3$ and $g \in W^{1, n}(\Omega)$ was proved in $[22,50,51]$ by using weighted estimates. Recently, Cellina [15] could relax the regularity assumption of $g \in W^{1,2}(\Omega)$ by adapting a Nirenberg technique. In the case $p \geq 3$ and $f \in W^{1, n}(\Omega)$ strictly bounded away from zero or satisfying certain growth condition, it has been shown that $u \in W_{l o c}^{2, q}(\Omega)$ for any $q<\frac{p-1}{p-2}$, see $[22,50,51]$.

Global (up to the boundary), full regularity for the second derivatives of the solutions of (4.1) with 0 boundary conditions are investigated in $[4,5,21,46]$. For $p \in(C(q), 2)$ and any bounded and sufficiently smooth domain $\Omega$, the authors proved $W^{2, q}$ regularity for any arbitrarily large $q$, getting as a by product result the Hölder continuity up to the boundary of the gradient of the solution for any $\alpha<1$. In particular, if $\Omega$ is convex, solutions belong to $W^{2,2}(\Omega)$ for any $1<p \leq 2$. The proofs are based on approximation arguments, the assumption that $p$ is close to 2 , and the classical Calderón-Zygmund theory. 
The restriction $p$ small is fundamental as the example of the functions $\left|x_{1}\right|^{\beta}$ with $\beta>1$ shows. These functions are local solutions to $-\Delta_{p} u=g$ for some $g \in L_{\text {loc }}^{\infty}\left(\mathbb{R}^{n}\right)$ provided that $p$ is large enough, but they fail to be in $W_{l o c}^{2,2}\left(\mathbb{R}^{n}\right)$ if $\beta \leq \frac{3}{2}$.

The Cordes condition for operators in nondivergence form. Here we recall some available results on the summability of the second derivative for operators in non-divergence form with measurable coefficients. The first are due to $[3,18,53]$ and require that the second order linear operator is close to the Laplacian. The case with lower order term was also treated in [17]. In [14], Campanato extended the $W^{2,2}$ estimate to $W^{2, q}$ for $q$ sufficiently close to 2. We refer the reader to [47, Theorem 1.2.1, Theorem 1.2.3] for a review on the Cordes condition.

Lemma 4.1. Consider a linear operator $L$ defined on the space $W_{l o c}^{2,2}(\Omega)$ as

$$
L v(x):=\sum_{i, j=1}^{n} a_{i, j}(x) D_{i} D_{j} v(x)
$$

where $a_{i j}(x)$ is a symmetric matrix with measurable coefficients satisfying the ellipticity condition

$$
\Lambda_{1}|\xi|^{2} \leq \sum_{i, j=1}^{n} a_{i, j}(x) \xi_{i} \xi_{j} \leq \Lambda_{2}|\xi|^{2}
$$

for some $0<\Lambda_{1}<\Lambda_{2}$ and satisfying the Cordes condition:

there exists $\delta \in(0,1]$ such that for a.e $x \in \Omega$

$$
\left(\sum_{i, j=1}^{n}\left(a_{i j}(x)\right)^{2}\right) \leq \frac{1}{n-1+\delta}\left(\sum_{i=1}^{n} a_{i i}(x)\right)^{2} .
$$

Then any strong solution $v \in W_{l o c}^{2,2}(\Omega) \cap L^{2}(\Omega)$ of the equation $L v=f$ in $\Omega$ with $f \in L^{2}(\Omega)$ satisfies, for any $\Omega^{\prime} \subset \subset \Omega$

$$
\int_{\Omega^{\prime}}\left|D^{2} v\right|^{2} d x \leq C\left(\|f\|_{L^{2}\left(\Omega^{\prime}\right)}^{2}+\|v\|_{L^{2}\left(\Omega^{\prime}\right)}^{2}\right)
$$

where $C=C\left(n, \Lambda_{1}, \Lambda_{2}, \delta, \Omega^{\prime}, \Omega\right)$.

Moreover, under the same hypothesis on the operator $L$, there exist two real numbers $1<q_{0}<2<q_{1}$ depending on the ellipticity constants and the dimension $n$, such that for any $q \in\left(q_{0}, q_{1}\right)$, any strong solution of $L v=f$ with $f \in L^{q}(\Omega)$, satisfies for any $\Omega^{\prime} \subset \subset \Omega$

$$
\int_{\Omega^{\prime}}\left|D^{2} v\right|^{q} d x \leq C\left(\|f\|_{L^{q}\left(\Omega^{\prime}\right)}^{q}+\|v\|_{L^{q}\left(\Omega^{\prime}\right)}^{q}\right)
$$

where $C=C\left(n, q, \Lambda_{1}, \Lambda_{2}, \delta, \Omega^{\prime}, \Omega\right)$. 
The Cordes condition is equivalent to the uniform ellipticity condition when $n=2$ and stronger when $n \geq 3$. As an application, it was used to prove the second order differentiability of $p$-harmonic functions in [45]. As it is often the case, the two dimensional case is an exception, since there is no restriction on $p$. We also mention the result of [42] which asserts that for uniformly elliptic linear equation with measurable coefficients, there exists a universal $r=r\left(\Lambda_{1}, \Lambda_{2}\right)>0$ such that any $C^{1,1}$ solution of $L v=f$ with $f \in L^{n}(\Omega)$ satisfies

$$
\|v\|_{W^{2, r}\left(\Omega^{\prime}\right)} \leq C\left(\|v\|_{L^{\infty}(\Omega)}+\|f\|_{L^{n}(\Omega)}\right),
$$

where $C=C\left(n, \Omega, \Lambda_{1}, \Lambda_{2}\right)>0$.

The second results can be found in [13] and relies on the smallness of the oscillation of the operator measured in the $L^{n}$ norm.

Lemma 4.2. Let $v$ be a bounded viscosity solution in $B_{1}$ of

$$
F\left(D^{2} v, x\right)=f(x) .
$$

Assume that $F$ is uniformly elliptic with ellipticity constants $\lambda$ and $\Lambda, F, f$ are continuous in $x, F(0, \cdot) \equiv 0$ and $F\left(D^{2} w, x_{0}\right)$ has $C^{1,1}$ interior estimates (with constant $c_{e}$ ) for any $x_{0}$ in $B_{1}$. Suppose that $f \in L^{q}(\Omega)$ for some $n<q<\infty$. Then there exist positive constants $\beta_{0}$ and $C$ depending on $n$, $\lambda, \Lambda, c_{e}$ and $q$, such that if the oscillation

$$
\beta\left(x, x_{0}\right):=\sup _{M \in \mathcal{S} \backslash\{0\}} \frac{\left|F(M, x)-F\left(M, x_{0}\right)\right|}{\|M\|}
$$

satisfies

$$
\left(\frac{\int_{B_{r}\left(x_{0}\right)} \beta\left(x, x_{0}\right)^{n} d x}{B_{r}\left(x_{0}\right)}\right)^{1 / n} \leq \beta_{0}
$$

for any ball $B_{r}\left(x_{0}\right) \subset B_{1}$, then $v \in W^{2, q}\left(B_{1 / 2}\right)$ and

$$
\|w\|_{W^{2, q}\left(B_{1 / 2}\right)} \leq C\left(\|w\|_{L^{\infty}\left(B_{1}\right)}+\|f\|_{L^{q}\left(B_{1}\right)}\right) .
$$

4.1. The case $\gamma \leq 0$ and $p$ close to 2. Using the result of Lemma 2.4, we can reduce the study to the case of the normalized $p$-Laplacian with a bounded right hand term. In this case the equation is singular but uniformly elliptic. We will thus use the result of Lemma 4.1 for regularized problems and provide uniform local $W^{2,2}$ estimates for $p$ in the range where Lemma 4.1 is satisfied, that is $1<p<3+\frac{2}{n-2}$.

Proof of Theorem 1.2. First of all, since for the moment, we can prove uniqueness only for $f=0$ (using the weak theory of the standard $p$-Laplacian) or $f$ with a constant sign (the proof is an adaptation of the arguments used in [36] using the viscosity theory), we use the classical trick which consists in adding an eigenvalue term in order to avoid dealing with the problem of uniqueness. 
Let $u$ be a viscosity solution of equation (1.1). For any $\lambda>0$, the function $u$ is a viscosity solution of

$$
-\Delta_{p}^{N} u(x)+\lambda u(x)=h(x):=f(x)|D u|^{-\gamma}+\lambda u(x), \quad x \in \Omega .
$$

Let $\Omega^{\prime} \subset \subset \Omega$ with $\Omega^{\prime}$ smooth enough. In the sequel we fix small enough $\lambda>0$ and a viscosity solution $u$ of (1.1). We take smooth functions $f_{\varepsilon} \in$ $C^{1}(\Omega) \cap L^{\infty}(\Omega)$ converging uniformly to $f$ in $\Omega^{\prime}$.

We consider the following regularized problem.

$$
\begin{cases}-\Delta v_{\varepsilon}-(p-2) \frac{D^{2} v_{\varepsilon} D v_{\varepsilon} \cdot D v_{\varepsilon}}{\left|D v_{\varepsilon}\right|^{2}+\varepsilon^{2}}+\lambda v_{\varepsilon}=f_{\varepsilon}\left(\left|D v_{\varepsilon}\right|^{2}+\varepsilon^{2}\right)^{-\gamma / 2}+\lambda u & \text { in } \Omega^{\prime} \\ v_{\varepsilon}=u & \text { on } \partial \Omega^{\prime} .\end{cases}
$$

It follows, using the fact that the problem is uniformly elliptic without singularities, solutions $v_{\varepsilon}$ are classical $C^{2}$ solutions and solve the equation in the classical sense, see [27, Theorem 15.18] and [37, Theorem 3.3]. From the comparison principle we have an estimate

$$
\left\|v_{\varepsilon}\right\|_{L^{\infty}\left(\Omega^{\prime}\right)} \leq 2\left(\|u\|_{L^{\infty}(\Omega)}+\frac{\|f\|_{L^{\infty}\left(\Omega^{\prime}\right)}}{\lambda}\right),
$$

In Appendix B we also show that for any $\Omega^{\prime \prime} \subset \subset \Omega^{\prime}$,

$$
\begin{aligned}
\left\|D v_{\varepsilon}\right\|_{L^{\infty}\left(\Omega^{\prime \prime}\right)} & \leq C\left(p, n, \gamma, \Omega^{\prime}\right)\left(\|f\|_{L^{\infty}\left(\Omega^{\prime}\right)}^{\frac{1}{1+\gamma}}+\left\|v_{\varepsilon}\right\|_{L^{\infty}\left(\Omega^{\prime}\right)}+\left\|h_{\varepsilon}\right\|_{L^{\infty}\left(\Omega^{\prime}\right)}\right) \\
& \leq C\left(p, n, \gamma, \Omega^{\prime}\right)\left(\|f\|_{L^{\infty}\left(\Omega^{\prime}\right)}^{\frac{1}{1+\gamma}}++\|u\|_{L^{\infty}\left(\Omega^{\prime}\right)}+\|f\|_{L^{\infty}\left(\Omega^{\prime}\right)}\right),
\end{aligned}
$$

where we denoted $h_{\varepsilon}:=\lambda\left(u-v_{\varepsilon}\right)$.

In the sequel we take $\lambda=1$. Consider the operator

$$
L_{v_{\varepsilon}}(v):=-\Delta v-(p-2) \frac{D^{2} v D v_{\varepsilon} \cdot D v_{\varepsilon}}{\left|D v_{\varepsilon}\right|^{2}+\varepsilon^{2}} .
$$

The operator $L_{v_{\varepsilon}}$ is uniformly elliptic with $\Lambda_{1}=\min (1, p-1)$ and $\Lambda_{2}=$ $\max (1, p-1)$ and satisfies the Cordes condition with $\delta=\delta(p, n)$ for $1<p<$ $3+\frac{2}{n-2}$. We have

$$
L_{v_{\varepsilon}} v_{\varepsilon}=F_{\varepsilon}
$$

where $F_{\varepsilon}:=h_{\varepsilon}+f_{\varepsilon}\left(\left|D v_{\varepsilon}\right|^{2}+\varepsilon^{2}\right)^{-\gamma / 2}$. Notice that $F_{\varepsilon}$ is uniformly bounded in $L^{2}\left(\Omega^{\prime \prime}\right)$. Indeed, for any $\Omega^{\prime \prime} \subset \subset \Omega^{\prime}$,

$$
\begin{aligned}
\left\|F_{\varepsilon}\right\|_{L^{2}\left(\Omega^{\prime}\right)} & =\left\|h_{\varepsilon}+f_{\varepsilon}\left(\left|D v_{\varepsilon}\right|^{2}+\varepsilon^{2}\right)^{-\gamma / 2}\right\|_{L^{2}\left(\Omega^{\prime \prime}\right)} \\
& \leq C(\gamma)\left(\left\|\left|D v_{\varepsilon}\right|^{-\gamma} f\right\|_{L^{2}\left(\Omega^{\prime \prime}\right)}+\|f\|_{L^{2}\left(\Omega^{\prime}\right)}\right)+\|u\|_{L^{2}\left(\Omega^{\prime}\right)}+\left\|v_{\varepsilon}\right\|_{L^{2}\left(\Omega^{\prime}\right)} \\
& \leq C(p, n, \gamma)\left|\Omega^{\prime}\right|^{1 / 2}\left(\|f\|_{L^{\infty}\left(\Omega^{\prime}\right)}^{\frac{1}{1+\gamma}}+\|f\|_{L^{\infty}\left(\Omega^{\prime}\right)}+\|u\|_{L^{\infty}\left(\Omega^{\prime}\right)}\right) .
\end{aligned}
$$


Lemma 4.1 implies that $D^{2} v_{\varepsilon}$ are uniformly bounded in $L_{l o c}^{2}\left(\Omega^{\prime}\right)$ and for $\Omega^{\prime \prime} \subset \subset \Omega^{\prime}$,

$$
\begin{aligned}
\int_{\Omega^{\prime \prime}}\left|D^{2} v_{\varepsilon}\right|^{2} d x & \leq C\left(\left\|F_{\varepsilon}\right\|_{L^{2}\left(\Omega^{\prime}\right)}+\left\|v_{\varepsilon}\right\|_{L^{2}(\Omega)}\right) \\
& \leq C(p, n, \gamma)\left|\Omega^{\prime}\right|^{1 / 2}\left(\|f\|_{L^{\infty}\left(\Omega^{\prime}\right)}^{\frac{1}{1+\gamma}}+\|f\|_{L^{\infty}\left(\Omega^{\prime}\right)}+\|u\|_{L^{\infty}\left(\Omega^{\prime}\right)}\right) .
\end{aligned}
$$

Moreover $\left\{v_{\varepsilon}\right\}$ are equi-Hölder continuous in $\overline{\Omega^{\prime}}$ since the boundary data is Hölder continuous and the operator is uniformly elliptic with ellipticity constants independent of $\varepsilon$, see [23, Theorem 1.2]. By the Arzelà-Ascoli theorem, $v_{\varepsilon} \rightarrow v_{\infty}$ uniformly in $\overline{\Omega^{\prime}}$. It follows from the relaxed limit stability that $v_{\infty}$ is a viscosity solution to

$$
-\Delta_{p}^{N} v_{\infty}+\lambda v_{\infty}=f\left|D v_{\infty}\right|^{-\gamma}+\lambda u
$$

with $v_{\infty}=u$ on $\partial \Omega^{\prime}$. By the uniqueness of viscosity solutions of (4.10) (see [23, Theorem 6.1] and [10, Proposition 2.2]), we conclude that $v_{\infty}=u$ and hence the estimate holds for $u$.

Notice that for $f \equiv 0$, using the result of Lemma 2.5 and the previous result, we have that for $\gamma>-1$ and $1<p<3+\frac{2}{n-2}$, any viscosity solution of $-|D u|^{\gamma} \Delta_{p}^{N} u=0$ belongs to $W_{l o c}^{2,2}(\Omega)$.

Note also that using a regularizing problem and using the result of Lemma 4.1, we can show that for $1<p<3+\frac{2}{n-2}, p-2 \leq q \leq p$ and $f \in L^{\infty}(\Omega)$, there exists a weak solution $u$ of $-\Delta_{p} u=f|D u|^{q}$ such that $u \in W_{l o c}^{2,2}(\Omega) \cap$ $C_{l o c}^{1, \alpha}(\Omega)$. This result extends the one stated in [38], where $q \geq p / 2$ and $1<p<3$.

Remark 4.3. In the case $\gamma<0, D$. Li and $Z$. Li $[39,40]$ studied regularity of viscosity solutions of

$$
-|D u|^{\gamma} F\left(D^{2} u, D u, u, x\right)=f
$$

where $F$ is uniformly elliptic. This equation includes equation (1.1). However, both their result and method are different from ours. They prove that there exists some $\delta>0$ depending on the ellipticity constants, dimension and $\gamma$, such that viscosity solutions of (4.11) are globally in $W^{2, \delta}(\Omega)$, and the estimate depends on the $L^{n}$-norm of $f$. Their method is based on an ABP estimate, barrier function method, touching by paraboloids, a localization argument, and a certain covering lemma, which playes a similar role than the standard Calderon-Zygmund cube decomposition lemma.

Remark 4.4. The classical results of fully non linear elliptic equations require a small oscillation condition on the coefficients. Applying the result of Lemma 4.2, we would also get uniform $W^{2, q}$ estimates for $p$ close to 2 (using that $\beta\left(x, x_{0}\right) \leq 2|p-2|$ ). Nevertheless, the results using the Cordes 
condition gives a more precise range on the values of $p$ where we are granted that $W^{2,2}$ estimates hold. In our case, since we have a uniform Lipschitz bound for $u$, Theorem 7.4 of [13] gives the existence of $\delta=\delta(p, n, \gamma)$ such that for every solution $u$ of (1.1) with $-1<\gamma \leq 0$ and $p>1$, we have $u \in W_{l o c}^{2, \delta}(\Omega)$, and

$$
\|u\|_{W^{2, \delta}\left(\Omega^{\prime}\right)} \leq C\left(p, n, \gamma, \Omega^{\prime}\right)\left(\|f\|_{L^{\infty}\left(\Omega^{\prime}\right)}^{\frac{1}{1+\gamma}}+\|u\|_{L^{\infty}\left(\Omega^{\prime}\right)}\right) .
$$

This provides an alternative proof for the result of Li and $\mathrm{Li}$ [39] in the special case of equation (1.1)

4.2. The case $\gamma>0$ but close to 0 and $p$ close to 2. In case that $\gamma>0$, we still have some results for $\gamma<1$ and $|p-2-\gamma|$ close to 0 . Consider smooth solutions to the following problem,

$$
\begin{cases}-\left(\left|D v_{\varepsilon}\right|^{2}+\varepsilon^{2}\right)^{\frac{\gamma}{2}}\left[\Delta v_{\varepsilon}-(p-2) \frac{D^{2} v_{\varepsilon} D v_{\varepsilon} \cdot D v_{\varepsilon}}{\left|D v_{\varepsilon}\right|^{2}+\varepsilon^{2}}\right]+\lambda v_{\varepsilon}=h_{\varepsilon} & \text { in } \Omega^{\prime} \\ v_{\varepsilon}=u & \text { on } \partial \Omega^{\prime}\end{cases}
$$

where $h_{\varepsilon}:=f_{\varepsilon}+\lambda u$, with $f_{\varepsilon}$ smooth and converging locally uniformly to $f$. The problem being uniformly elliptic without singularities and the right hand side being $C^{1}$-continuous, the function $v_{\varepsilon}$ are in $C^{2, \alpha}$. The existence of smooth solutions $v_{\varepsilon}$ is ensured by the classical theory (see [27, Theorem $15.18])$.

Proof of Theorem 1.3.

Step 1: Uniform Lipschitz estimates.

Applying the comparison principle for elliptic quasilinear equation in general form (see [27, Theorem 10,1]), we have that

$$
\begin{aligned}
\left\|v_{\varepsilon}\right\|_{L^{\infty}\left(\Omega^{\prime}\right)} & \leq C\left(\|u\|_{L^{\infty}(\Omega)}+\frac{\left\|h_{\varepsilon}\right\|_{L^{\infty}\left(\Omega^{\prime}\right)}}{\lambda}\right) \\
& \leq C\left(\|u\|_{L^{\infty}(\Omega)}+\frac{\|f\|_{L^{\infty}\left(\Omega^{\prime}\right)}}{\lambda}\right) .
\end{aligned}
$$

Next, if $\gamma \leq p-2$ then $v_{\varepsilon}$ are solutions to

$$
-\operatorname{div}\left(\left(\left|D v_{\varepsilon}\right|^{2}+\varepsilon^{2}\right)^{\frac{p-2}{2}} D v_{\varepsilon}\right)=\left(h_{\varepsilon}-\lambda v_{\varepsilon}\right)\left(\left|D v_{\varepsilon}\right|^{2}+\varepsilon^{2}\right)^{\frac{p-2-\gamma}{2}} .
$$

Using that the boundary data $u$ is in $C^{1, \alpha}\left(\overline{\Omega^{\prime}}\right)$, it follows from [41, Theorem 1] that $v_{\varepsilon}$ are uniformly bounded in $C^{1, \alpha}\left(\overline{\Omega^{\prime}}\right)$. We also have a uniform Lipschitz bound on $v_{\varepsilon}$ using the Ishii-Lions method in the case where we lack the divergence structure $(\gamma>p-2)$

$$
\left\|D v_{\varepsilon}\right\|_{L^{\infty}\left(\Omega^{\prime \prime}\right)} \leq C(n, p, \gamma)\left(\|h\|_{L^{\infty}\left(\Omega^{\prime}\right)}+\left\|v_{\varepsilon}\right\|_{L^{\infty}\left(\Omega^{\prime}\right)}\right) .
$$

The proof of this estimate is provided in the appendix, see Proposition B.2. 
Step 2: Uniform estimates for the Hessian.

Equation (4.12) can be regarded as a perturbation of the regularized $\gamma$ Laplacian. Indeed, we can rewrite

$$
\begin{aligned}
& -\left(\left|D v_{\varepsilon}\right|^{2}+\varepsilon^{2}\right)^{\frac{\gamma}{2}}\left[\Delta v_{\varepsilon}+(p-2) \frac{D^{2} v_{\varepsilon} D v_{\varepsilon} \cdot D v_{\varepsilon}}{\left|D v_{\varepsilon}\right|^{2}+\varepsilon^{2}}\right] \\
& =-\operatorname{div}\left(\left(\left|D v_{\varepsilon}\right|^{2}+\varepsilon^{2}\right)^{\frac{\gamma}{2}} D v_{\varepsilon}\right)-(p-2-\gamma)\left(\left|D v_{\varepsilon}\right|^{2}+\varepsilon^{2}\right)^{\frac{\gamma}{2}} \frac{D^{2} v_{\varepsilon} D v_{\varepsilon} \cdot D v_{\varepsilon}}{\left|D v_{\varepsilon}\right|^{2}+\varepsilon^{2}} \\
& =-\frac{p-2}{\gamma} \operatorname{div}\left(\left(\left|D v_{\varepsilon}\right|^{2}+\varepsilon^{2}\right)^{\frac{\gamma}{2}} D v_{\varepsilon}\right)+\frac{p-2-\gamma}{\gamma}\left(\left|D v_{\varepsilon}\right|^{2}+\varepsilon^{2}\right)^{\frac{\gamma}{2}} \Delta v_{\varepsilon} .
\end{aligned}
$$

Hence we have

$-\operatorname{div}\left(\left(\left|D v_{\varepsilon}\right|^{2}+\varepsilon^{2}\right)^{\frac{\gamma}{2}} D v_{\varepsilon}\right)=(p-2-\gamma)\left(\left|D v_{\varepsilon}\right|^{2}+\varepsilon^{2}\right)^{\frac{\gamma}{2}} \frac{D^{2} v_{\varepsilon} D v_{\varepsilon} \cdot D v_{\varepsilon}}{\left|D v_{\varepsilon}\right|^{2}+\varepsilon^{2}}+g_{\varepsilon}$,

where $g_{\varepsilon}:=f_{\varepsilon}+\lambda u-\lambda v_{\varepsilon}$. Multiplying by a smooth test function $\phi \in C_{c}^{2}\left(\Omega^{\prime}\right)$ and integrating by parts, we have

$$
\begin{aligned}
\int_{\Omega^{\prime}} & \left(\left|D v_{\varepsilon}\right|^{2}+\varepsilon^{2}\right)^{\frac{\gamma}{2}} D v_{\varepsilon} \cdot D \phi d x \\
& =\int_{\Omega^{\prime}}\left((p-2-\gamma)\left(\left|D v_{\varepsilon}\right|^{2}+\varepsilon^{2}\right)^{\frac{\gamma}{2}} \frac{D^{2} v_{\varepsilon} D v_{\varepsilon} \cdot D v_{\varepsilon}}{\left|D v_{\varepsilon}\right|^{2}+\varepsilon^{2}}+g_{\varepsilon}\right) \phi d x .
\end{aligned}
$$

Choosing $D_{s} \phi$ instead of $\phi$ for $s \in\{1, \ldots, n\}$ as a test function and integrating by parts, we get

$$
\begin{aligned}
& \int_{\Omega^{\prime}}\left(\left|D v_{\varepsilon}\right|^{2}+\varepsilon^{2}\right)^{\frac{\gamma}{2}}\left[\sum_{i} D_{i} D_{s} v_{\varepsilon} D_{i} \phi+\gamma \sum_{i, j} \frac{D_{j} v_{\varepsilon} D_{j} D_{s} v_{\varepsilon} D_{i} v_{\varepsilon} D_{i} \phi}{\left|D v_{\varepsilon}\right|^{2}+\varepsilon^{2}}\right] d x \\
& =-\int_{\Omega^{\prime}} D_{s} \phi\left((p-2-\gamma)\left(\left|D v_{\varepsilon}\right|^{2}+\varepsilon^{2}\right)^{\frac{\gamma}{2}} \frac{D^{2} v_{\varepsilon} D v_{\varepsilon} \cdot D v_{\varepsilon}}{\left|D v_{\varepsilon}\right|^{2}+\varepsilon^{2}}+g_{\varepsilon}\right) d x \\
& =-\int_{\Omega^{\prime}} D_{s} \phi(p-2-\gamma)\left(\left|D v_{\varepsilon}\right|^{2}+\varepsilon^{2}\right)^{\frac{\gamma}{2}} \frac{D^{2} v_{\varepsilon} D v_{\varepsilon} \cdot D v_{\varepsilon}}{\left|D v_{\varepsilon}\right|^{2}+\varepsilon^{2}} d x+\int_{\Omega^{\prime}} \phi D_{s} g_{\varepsilon} d x .
\end{aligned}
$$

Denoting

$$
\tilde{A}^{\varepsilon}:=I d+\gamma \frac{D v_{\varepsilon} \otimes D v_{\varepsilon}}{\left|D v_{\varepsilon}\right|^{2}+\varepsilon^{2}}
$$

and

$$
H\left(D v_{\varepsilon}\right):=\left(\left|D v_{\varepsilon}\right|^{2}+\varepsilon^{2}\right)^{\frac{1}{2}}
$$

we have

$$
\begin{aligned}
& \int_{\Omega^{\prime}} H\left(D v_{\varepsilon}\right)^{\gamma} \sum_{i, j} \tilde{A}_{i j}^{\varepsilon} D_{j} D_{s} v_{\varepsilon} D_{i} \phi d x \\
& =-(p-2-\gamma) \int_{\Omega^{\prime}} D_{s} \phi H\left(D v_{\varepsilon}\right)^{\gamma} \frac{D^{2} v_{\varepsilon} D v_{\varepsilon} \cdot D v_{\varepsilon}}{\left|D v_{\varepsilon}\right|^{2}+\varepsilon^{2}} d x+\int_{\Omega^{\prime}} \phi D_{s} g_{\varepsilon} d x .
\end{aligned}
$$


For $0<\beta<1$, taking

$$
\phi:=\eta^{2}\left(\left|D v_{\varepsilon}\right|^{2}+\varepsilon^{2}\right)^{\frac{-\beta}{2}} D_{s} v_{\varepsilon}
$$

where $\eta \in C_{c}^{\infty}$ is a non negative cut-off function, we have

$$
\begin{aligned}
D_{i} \phi= & \eta^{2} H\left(D v_{\varepsilon}\right)^{-\beta} D_{i} D_{s} v_{\varepsilon}+2 \eta D_{i} \eta H\left(D v_{\varepsilon}\right)^{-\beta} D_{s} v_{\varepsilon} \\
& -\beta \eta^{2} \sum_{l} D_{i} D_{l} v_{\varepsilon} D_{l} v_{\varepsilon} H\left(D v_{\varepsilon}\right)^{-\beta-2} D_{s} v_{\varepsilon} .
\end{aligned}
$$

Summing up over $s \in\{1, \ldots, n\}$, it follows that

$$
\begin{aligned}
& I_{1}+I_{2}+I_{3}:=\int_{\Omega^{\prime}} \eta^{2} H\left(D v_{\varepsilon}\right)^{\gamma-\beta} \sum_{i, j, s}\left(\tilde{A}_{i j}^{\varepsilon} D_{j} D_{s} v_{\varepsilon} D_{i} D_{s} v_{\varepsilon}\right) d x \\
& -\beta \int_{\Omega^{\prime}} \eta^{2} H\left(D v_{\varepsilon}\right)^{\gamma-\beta-2} \sum_{i, j, s}\left(\tilde{A}_{i j}^{\varepsilon} D_{j} D_{s} v_{\varepsilon} D_{s} v_{\varepsilon} \sum_{l} D_{i} D_{l} v_{\varepsilon} D_{l} v_{\varepsilon}\right) d x \\
& +\int_{\Omega^{\prime}} 2 \eta H\left(D v_{\varepsilon}\right)^{\gamma-\beta} \sum_{i, j, s}\left(\tilde{A}_{i j}^{\varepsilon} D_{j} D_{s} v_{\varepsilon} D_{s} v_{\varepsilon}\right) D_{i} \eta d x \\
= & -(p-2-\gamma) \int_{\Omega^{\prime}} \eta^{2} \sum_{s} D_{s} D_{s} v_{\varepsilon} H\left(D v_{\varepsilon}\right)^{\gamma-\beta} \frac{D^{2} v_{\varepsilon} D v_{\varepsilon} \cdot D v_{\varepsilon}}{\left|D v_{\varepsilon}\right|^{2}+\varepsilon^{2}} d x \\
& +\beta(p-2-\gamma) \int_{\Omega^{\prime}} \eta^{2} \sum_{s, l} D_{s} v_{\varepsilon} D_{s} D_{l} v_{\varepsilon} D_{l} v_{\varepsilon} H\left(D v_{\varepsilon}\right)^{\gamma-\beta-2} \frac{D^{2} v_{\varepsilon} D v_{\varepsilon} \cdot D v_{\varepsilon}}{\left|D v_{\varepsilon}\right|^{2}+\varepsilon^{2}} d x \\
& -2(p-2-\gamma) \int_{\Omega^{\prime}} \eta \sum_{s} D_{s} \eta D_{s} v_{\varepsilon} H\left(D v_{\varepsilon}\right)^{\gamma-\beta} \frac{D^{2} v_{\varepsilon} D v_{\varepsilon} \cdot D v_{\varepsilon}}{\left|D v_{\varepsilon}\right|^{2}+\varepsilon^{2}} d x \\
& +\int_{\Omega^{\prime}} \sum_{s} D_{s} g_{\varepsilon} D_{s} v_{\varepsilon} \eta^{2} H\left(D v_{\varepsilon}\right)^{-\beta} d x \\
= & I I_{1}+I I_{2}+I I_{3}+I I_{4} .
\end{aligned}
$$

That is,

$$
I_{1}+I_{2}=I I_{1}+I I_{2}+I I_{3}+I I_{4}-I_{3} .
$$

The estimates of $I_{1}, I_{2}, I_{3}$ are given as follows. We have

$$
\begin{aligned}
I_{1}+I_{2} & =\int_{\Omega^{\prime}} \eta^{2} H\left(D v_{\varepsilon}\right)^{\gamma-\beta}\left[\left|D^{2} v_{\varepsilon}\right|^{2}-\frac{\beta}{4}\left|D\left(\left|D v_{\varepsilon}\right|^{2}\right)\right|^{2} H\left(D v_{\varepsilon}\right)^{-2}\right] d x \\
& +\underbrace{\frac{\gamma}{4} \int_{\Omega^{\prime}} \eta^{2} H\left(D v_{\varepsilon}\right)^{\gamma-\beta-2}\left[\left|D\left(\left|D v_{\varepsilon}\right|^{2}\right)\right|^{2}-\beta\left|D v_{\varepsilon} \cdot D\left(\left|D v_{\varepsilon}\right|^{2} \mid\right)\right|^{2} H^{-1}\right] d x}_{\geq 0}
\end{aligned}
$$


so that for $\gamma>0$

$$
\begin{aligned}
I_{1}+I_{2} \geq & (1-\beta) \int_{\Omega^{\prime}} \eta^{2} H\left(D v_{\varepsilon}\right)^{\gamma-\beta}\left|D^{2} v_{\varepsilon}\right|^{2} d x \\
& +\frac{(1-\beta) \gamma}{4} \int_{\Omega^{\prime}} \eta^{2} H\left(D v_{\varepsilon}\right)^{\gamma-\beta-2}\left|D\left(\left|D v_{\varepsilon}\right|^{2}\right)\right|^{2} d x .
\end{aligned}
$$

Next, using Young's inequality, it follows that

$$
\begin{aligned}
\left|2 H\left(D v_{\varepsilon}\right)^{\gamma-\beta} \eta \sum_{i, j, s}\left(\tilde{A}_{i j}^{\varepsilon} D_{j} D_{s} v_{\varepsilon} D_{s} v_{\varepsilon}\right) D_{i} \eta\right| & \leq \delta_{1}\left|D\left(\left|D v_{\varepsilon}\right|^{2}\right)\right|^{2} \eta^{2} H\left(D v_{\varepsilon}\right)^{\gamma-\beta-2} \\
& +C\left(p, \delta_{1}\right)|D \eta|^{2} H\left(D v_{\varepsilon}\right)^{\gamma-\beta+2}
\end{aligned}
$$

and hence

$$
\begin{aligned}
\left|I_{3}\right| & \leq \delta_{1} \int_{\Omega^{\prime}}\left|D\left(\left|D v_{\varepsilon}\right|^{2}\right)\right|^{2} \eta^{2} H\left(D v_{\varepsilon}\right)^{\gamma-\beta-2} d x \\
& +C\left(p, \delta_{1}\right) \int_{\Omega^{\prime}}|D \eta|^{2} H\left(D v_{\varepsilon}\right)^{\gamma-\beta+2} d x .
\end{aligned}
$$

To estimate the terms $I I_{1}$ and $I I_{2}$, we will use that $\left|D^{2} v_{\varepsilon} D v_{\varepsilon}, D v_{\varepsilon}\right| \leq$ $\left|D^{2} v_{\varepsilon}\right|\left|D v_{\varepsilon}\right|^{2}$ and $\left|\Delta v_{\varepsilon}\right| \leq \sqrt{n}\left|D^{2} v_{\varepsilon}\right|$. We obtain

$$
\begin{aligned}
& \left|I I_{1}\right| \leq \int_{\Omega^{\prime}}|p-2-\gamma| \sqrt{n}\left|D^{2} v\right|^{2} \eta^{2} H\left(D v_{\varepsilon}\right)^{\gamma-\beta} d x \\
& \left|I I_{2}\right| \leq \beta(p-2-\gamma)^{+} \int_{\Omega^{\prime}}\left|D^{2} v\right|^{2} \eta^{2} H\left(D v_{\varepsilon}\right)^{\gamma-\beta} d x .
\end{aligned}
$$

Using Young's inequality, we can estimate $I I_{3}$ by

$$
\left|I I_{3}\right| \leq\left.\left.\delta_{2} \int_{\Omega^{\prime}}|D| D v_{\varepsilon}\right|^{2}\right|^{2} \eta^{2} H\left(D v_{\varepsilon}\right)^{\gamma-\beta} d x+C\left(p, \delta_{2}, \gamma\right) \int_{\Omega^{\prime}}|D \eta|^{2} H\left(D v_{\varepsilon}\right)^{\gamma-\beta+2} d x .
$$

Finally we estimate $I I_{4}$ by

$$
\left|I I_{4}\right| \leq \int_{\Omega^{\prime}}|| H\left(D v_{\varepsilon}\right)||_{L^{\infty}\left(\Omega^{\prime}\right)}^{1-\beta} \eta^{2}\left|D g_{\varepsilon}\right| d x
$$

Choosing $\delta_{1}$ and $\delta_{2}$ small enough, such that

$$
(1-\beta) \frac{\gamma}{4}-\delta_{1}-\delta_{2}=0
$$

we obtain 


$$
\begin{aligned}
(1-\beta) \int_{\Omega^{\prime}} \eta^{2} H\left(D v_{\varepsilon}\right)^{\gamma-\beta}\left|D^{2} v_{\varepsilon}\right|^{2} & \leq \beta(p-2-\gamma)^{+} \int_{\Omega^{\prime}}\left|D^{2} v\right|^{2} \eta^{2} H\left(D v_{\varepsilon}\right)^{\gamma-\beta} d x \\
& +\int_{\Omega^{\prime}}|p-2-\gamma| \sqrt{n}\left|D^{2} v_{\varepsilon}\right|^{2} \eta^{2} H\left(D v_{\varepsilon}\right)^{\gamma-\beta} d x \\
& +\left.\int_{\Omega^{\prime}}|| H\left(D v_{\varepsilon}\right)\right|_{L^{\infty}\left(\Omega^{\prime}\right)} ^{1-\beta} \eta^{2}\left|D g_{\varepsilon}\right| d x \\
& +\int_{\Omega^{\prime}} C(p, n, \gamma)|D \eta|^{2} H\left(D v_{\varepsilon}\right)^{\gamma-\beta+2} d x .
\end{aligned}
$$

Now, if

$$
1-\beta-\sqrt{n}|p-2-\gamma|-\beta(p-2-\gamma)^{+}=\kappa>0,
$$

we get

$$
\begin{aligned}
\kappa \int_{\Omega^{\prime}} \eta^{2} H\left(D v_{\varepsilon}\right)^{\gamma-\beta}\left|D^{2} v_{\varepsilon}\right|^{2} & \leq \int_{\Omega^{\prime}}|| H\left(D v_{\varepsilon}\right)||_{L^{\infty}\left(\Omega^{\prime}\right)}^{1-\beta} \eta^{2}\left|D g_{\varepsilon}\right| d x \\
& +\int_{\Omega^{\prime}} C(p, n, \gamma)|D \eta|^{2} H\left(D v_{\varepsilon}\right)^{\gamma-\beta+2} d x
\end{aligned}
$$

We fix $\lambda=1$. By using (4.13) and (4.14), we have

$$
H\left(D v_{\varepsilon}\right) \leq C(n, p, \gamma)\left(\|u\|_{L^{\infty}\left(\Omega^{\prime}\right)}+\|f\|_{L^{\infty}\left(\Omega^{\prime}\right)}\right)
$$

and

$$
\begin{aligned}
\int_{\Omega^{\prime}}\left|D g_{\varepsilon}\right| d x & =\int_{\Omega^{\prime}}\left|D f_{\varepsilon}+\lambda D u-\lambda D v_{\varepsilon}\right| d x \\
& \leq \int_{\Omega^{\prime}}|D f| d x+\lambda\left|\Omega^{\prime}\right|\left(\|D u\|_{L^{\infty}\left(\Omega^{\prime}\right)}+\left\|D v_{\varepsilon}\right\|_{L^{\infty}\left(\Omega^{\prime}\right)}\right) \\
& \leq C\left(p, n, \gamma,\left|\Omega^{\prime}\right|\right)\left(\|f\|_{W^{1,1}\left(\Omega^{\prime}\right)}+\|u\|_{L^{\infty}\left(\Omega^{\prime}\right)}+\|f\|_{L^{\infty}\left(\Omega^{\prime}\right)}\right)
\end{aligned}
$$

We take $\eta$ such that $\eta \equiv 1$ on $\Omega^{\prime \prime} \subset \subset \Omega^{\prime}, 0 \leq \eta \leq 1$ and $\|D \eta\|_{L^{\infty}\left(\Omega^{\prime}\right)} \leq C$. For any $\beta \in(0,1)$ and $0<\gamma \leq \beta$, assuming that (4.17) holds and $f \in$ $W^{1,1}(\Omega) \cap C(\Omega)$, we get that $D^{2} v_{\varepsilon}$ is uniformly bounded in $L^{2}\left(\Omega^{\prime \prime}\right)$,

$$
\int_{\Omega^{\prime \prime}}\left|D^{2} v_{\varepsilon}\right|^{2} d x \leq C\left(n, p, \gamma, \beta, d, d^{\prime},\|f\|_{W^{1,1}\left(\Omega^{\prime}\right)},\|f\|_{L^{\infty}\left(\Omega^{\prime}\right)},\|u\|_{L^{\infty}\left(\Omega^{\prime}\right)}\right) .
$$

If $\gamma \geq 0$ and $p>1$, then using Proposition B.1 below together with [28, Theorem 1], we see that $v_{\varepsilon}$ are uniformly bounded in $C^{\bar{\alpha}}\left(\bar{\Omega}^{\prime}\right.$ ) (it is easy to check that the condition of [28, Theorem 1] are satisfied with constants independent of $\varepsilon$ ). It follows from the Arzelà-Ascoli theorem, that up to a subsequence $v_{\varepsilon}$ converges to some function $v$. Using the stability result, we have that $v$ is a viscosity solution of

$$
-|D v|^{\gamma} \Delta_{p}^{N} v+\lambda v=f+\lambda u
$$


in $\Omega^{\prime}$ with $v=u$ on $\partial \Omega^{\prime}$. By uniqueness of solutions of (4.19) for $\lambda>0$ (see [6, Theorem 1.1]), we conclude that $u=v$ in $\Omega^{\prime}$. Passing to the limit in (4.18), we get the desired local $W^{2,2}$-estimate for $u$, so the proof of Theorem 1.3 is complete.

Remark 4.5. For $\gamma=p-2,2<p<3$ and $f \in W_{\text {loc }}^{1,1}(\Omega) \cap C(\Omega)$, we recover the $W_{\text {loc }}^{2,2}(\Omega)$ regularity for weak solutions of the p-Poisson problem $-\Delta_{p} u=f$.

\section{Appendix A. Proof of Lemma 3.1}

In the proof we denote by $S^{n}$ the set of symmetric $n \times n$ matrices. For $\eta, \xi \in \mathbb{R}^{n}$, we denote by $\eta \otimes \xi$ the $n \times n$-matrix for which $(\eta \otimes \xi)_{i j}=\eta_{i} \xi_{j}$. For $n \times n$ matrices we use the matrix norm

$$
\|A\|:=\sup _{|x| \leq 1}\{|A x|\}
$$

Proof of Lemma 3.1 We use the viscosity method introduced by Ishii and Lions in [32].

We are going to show that $\tilde{w}$ is Hölder in $B_{3 / 4}$, and this will imply that $\tilde{w}$ is Hölder in any smaller ball $B_{\rho}$ for $\rho \in\left(0, \frac{3}{4}\right)$ with the same Hölder constant. We fix $x_{0} \in B_{\frac{3}{4}}$, and consider the auxiliary function

$$
\Phi(x, y):=\tilde{w}(x)-\tilde{w}(y)-L \phi(|x-y|)-\frac{M}{2}\left|x-x_{0}\right|^{2}-\frac{M}{2}\left|y-x_{0}\right|^{2},
$$

where $\phi(t)=t^{\beta}$ for some $0<\beta<1$. Our goal is to show that $\Phi(x, y) \leq 0$ for $(x, y) \in B_{r} \times B_{r}$, where $r=\frac{6}{7}$. We will argue by contradiction, write two viscosity inequalities and combine them in order to get a contradiction using the second order terms and the strict ellipticity in the gradient direction.

We argue by contradiction and assume that for all $L>0, M>0$ and $\beta \in(0,1), \Phi$ has a positive maximum. We assume that the maximum is reached at some point $\left(x_{1}, y_{1}\right) \in \bar{B}_{r} \times \bar{B}_{r}$. Since $\tilde{w}$ is continuous and bounded, we get

$$
\begin{aligned}
& M\left|x_{1}-x_{0}\right|^{2} \leq 2\|\tilde{w}\|_{L^{\infty}\left(B_{1}\right)}, \\
& M\left|y_{1}-x_{0}\right|^{2} \leq 2\|\tilde{w}\|_{L^{\infty}\left(B_{1}\right)} .
\end{aligned}
$$

Notice that $x_{1} \neq y_{1}$, otherwise the maximum of $\Phi$ would be non positive. Taking $M \geq\|\tilde{w}\|_{L^{\infty}\left(B_{1}\right)}\left(\frac{32}{r}\right)^{2}$, we have $\left|x_{1}-x_{0}\right|<r / 8$ and $\left|y_{1}-x_{0}\right|<r / 8$ so that $x_{1}$ and $y_{1}$ are in $B_{r}$.

Next we apply Jensen-Ishii's lemma to $\tilde{w}(x)-\frac{M}{2}\left|x-x_{0}\right|^{2}$ and $\tilde{w}(y)+$ $\frac{M}{2}\left|y-x_{0}\right|^{2}$. By Jensen-Ishii's lemma (also known as theorem of sums, see 
[20, Theorem 3.2]), there exist

$$
\begin{aligned}
& \left(\zeta_{x}, X\right) \in \overline{\mathcal{J}}^{2,+}\left(\tilde{w}\left(x_{1}\right)-\frac{M}{2}\left|x_{1}-x_{0}\right|^{2}\right), \\
& \left(\zeta_{y}, Y\right) \in \overline{\mathcal{J}}^{2,-}\left(\tilde{w}\left(y_{1}\right)+\frac{M}{2}\left|y_{1}-x_{0}\right|^{2}\right),
\end{aligned}
$$

that is

$$
\begin{aligned}
& (a, X+M I) \in \overline{\mathcal{J}}^{2,+} \tilde{w}\left(x_{1}\right), \\
& (b, Y-M I) \in \overline{\mathcal{J}}^{2,-} \tilde{w}\left(y_{1}\right),
\end{aligned}
$$

where $\left(\zeta_{x}=\zeta_{y}\right)$

$$
\begin{gathered}
a=L \phi^{\prime}\left(\left|x_{1}-y_{1}\right|\right) \frac{x_{1}-y_{1}}{\left|x_{1}-y_{1}\right|}+M\left(x_{1}-x_{0}\right)=\zeta_{x}+M\left(x_{1}-x_{0}\right), \\
b=L \phi^{\prime}\left(\left|x_{1}-y_{1}\right|\right) \frac{x_{1}-y_{1}}{\left|x_{1}-y_{1}\right|}-M\left(y_{1}-x_{0}\right)=\zeta_{y}-M\left(y_{1}-x_{0}\right) .
\end{gathered}
$$

If $L$ is large enough (depending on $M$, actually since $\left|x_{1}-x_{0}\right| \leq 2,\left|y_{1}-y_{0}\right| \leq$ $2,\left|x_{1}-y_{1}\right| \leq 2$, it is enough that $L>\frac{M}{\beta} 2^{4-\beta}$ ), we have

$$
\begin{aligned}
& 2 L \beta\left|x_{1}-y_{1}\right|^{\beta-1} \geq|a| \geq L \phi^{\prime}\left(\left|x_{1}-y_{1}\right|\right)-M\left|x_{1}-x_{0}\right| \geq \frac{L}{2} \beta\left|x_{1}-y_{1}\right|^{\beta-1} . \\
& 2 L \beta\left|x_{1}-y_{1}\right|^{\beta-1} \geq|b| \geq L \phi^{\prime}\left(\left|x_{1}-y_{1}\right|\right)-M\left|y_{1}-y_{0}\right| \geq \frac{L}{2} \beta\left|x_{1}-y_{1}\right|^{\beta-1} .
\end{aligned}
$$

Moreover, by Jensen-Ishii's lemma [19], for any $\tau>0$, we can take $X, Y \in \mathcal{S}^{n}$ such that for all $\tau>0$ such that $\tau B<I$, we have

$$
-\frac{2}{\tau}\left(\begin{array}{cc}
I & 0 \\
0 & I
\end{array}\right) \leq\left(\begin{array}{cc}
X & 0 \\
0 & -Y
\end{array}\right) \leq\left(\begin{array}{cc}
B^{\tau} & -B^{\tau} \\
-B^{\tau} & B^{\tau}
\end{array}\right)
$$

where

$$
\begin{aligned}
B= & L \phi^{\prime \prime}\left(\left|x_{1}-y_{1}\right|\right) \frac{x_{1}-y_{1}}{\left|x_{1}-y_{1}\right|} \otimes \frac{x_{1}-y_{1}}{\left|x_{1}-y_{1}\right|} \\
& +\frac{L \phi^{\prime}\left(\left|x_{1}-y_{1}\right|\right)}{\left|x_{1}-y_{1}\right|}\left(I-\frac{x_{1}-y_{1}}{\left|x_{1}-y_{1}\right|} \otimes \frac{x_{1}-y_{1}}{\left|x_{1}-y_{1}\right|}\right) \\
= & L \beta\left|x_{1}-y_{1}\right|^{\beta-2}\left(I+(\beta-2) \frac{x_{1}-y_{1}}{\left|x_{1}-y_{1}\right|} \otimes \frac{x_{1}-y_{1}}{\left|x_{1}-y_{1}\right|}\right)
\end{aligned}
$$

and

$$
B^{\tau}=(I-\tau B)^{-1} B
$$

For $\tau=\frac{1}{2 L \beta\left|x_{1}-y_{1}\right|^{\beta-2}}$, we have

$B^{\tau}=(I-\tau B)^{-1} B=2 L \beta\left|x_{1}-y_{1}\right|^{\beta-2}\left(I-2 \frac{2-\beta}{3-\beta} \frac{x_{1}-y_{1}}{\left|x_{1}-y_{1}\right|} \otimes \frac{x_{1}-y_{1}}{\left|x_{1}-y_{1}\right|}\right)$.

Notice that for $\xi=\frac{x_{1}-y_{1}}{\left|x_{1}-y_{1}\right|}$, we have 


$$
\left\langle B^{\tau} \xi, \xi\right\rangle=2 L \beta\left|x_{1}-y_{1}\right|^{\beta-2}\left(\frac{\beta-1}{3-\beta}\right)<0 .
$$

Applying the inequality (A.2) to any vector $(\xi, \xi)$ with $|\xi|=1$, we have that $X-Y \leq 0$ and

$$
\|X\|,\|Y\| \leq 2 L \beta\left|x_{1}-y_{1}\right|^{\beta-2} .
$$

The reader can find more details in $[19,20,32]$.

Denoting $\eta_{1}=a+e, \eta_{2}=b+e$, we have for $L$ large enough (it is enough that $L>\frac{4}{\beta} 2^{1-\beta} \geq \frac{4}{\beta}\left|x_{1}-y_{1}\right|^{1-\beta}$ )

$$
\begin{aligned}
& 3 L \beta\left|x_{1}-y_{1}\right|^{\beta-1} \geq\left|\eta_{1}\right| \geq|a|-1 \geq \frac{|a|}{2} \geq \frac{L}{4} \beta\left|x_{1}-y_{1}\right|^{\beta-1}, \\
& 3 L \beta\left|x_{1}-y_{1}\right|^{\beta-1} \geq\left|\eta_{2}\right| \geq|b|-1 \geq \frac{|b|}{2} \geq \frac{L}{4} \beta\left|x_{1}-y_{1}\right|^{\beta-1} .
\end{aligned}
$$

The viscosity inequalities read as

$$
\begin{aligned}
& -f\left(x_{1}\right)|q|^{-\gamma-1} \leq\left|\eta_{1}\right|^{\gamma}\left[\operatorname{tr}(X+M I)+(p-2) \frac{\langle(X+M I)(a+e),(a+e)\rangle}{|a+e|^{2}}\right], \\
& -f\left(y_{1}\right)|q|^{-\gamma-1} \geq\left|\eta_{2}\right|^{\gamma}\left[\operatorname{tr}(Y-M I)+(p-2) \frac{\langle(Y-M I)(b+e),(b+e)\rangle}{|b+e|^{2}}\right] .
\end{aligned}
$$

In other words,

$$
\begin{aligned}
-f\left(x_{1}\right)|q|^{-\gamma-1}\left|\eta_{1}\right|^{-\gamma} & \leq \operatorname{tr}\left(A\left(\eta_{1}\right)(X+M I)\right) \\
f\left(y_{1}\right)|q|^{-\gamma-1}\left|\eta_{2}\right|^{-\gamma} & \leq-\operatorname{tr}\left(A\left(\eta_{2}\right)(Y-M I)\right)
\end{aligned}
$$

where for $\eta \neq 0 \bar{\eta}=\frac{\eta}{|\eta|}$ and

$$
A(\eta):=I+(p-2) \bar{\eta} \otimes \bar{\eta} .
$$

Adding the two inequalities, we obtain $|q|^{-\gamma-1}\left[f\left(y_{1}\right)\left|\eta_{2}\right|^{-\gamma}-f\left(x_{1}\right)\left|\eta_{1}\right|^{-\gamma}\right] \leq \operatorname{tr}\left(A\left(\eta_{1}\right)(X+M I)\right)-\operatorname{tr}\left(A\left(\eta_{2}\right)(Y-M I)\right)$. It results (using that $\left|\eta_{i}\right|^{-\gamma} \leq C(\beta)\left(L\left|x_{1}-y_{1}\right|^{\beta-1}\right)^{-\gamma}$ ) that

$$
\begin{aligned}
-C(\beta) 2|q|^{-\gamma-1}\|f\|_{L^{\infty}\left(B_{1}\right)}\left(L\left|x_{1}-y_{1}\right|^{\beta-1}\right)^{-\gamma} \leq & \underbrace{\operatorname{tr}\left(A\left(\eta_{1}\right)(X-Y)\right)}_{(I)} \\
& +\underbrace{\operatorname{tr}\left(\left(A\left(\eta_{1}\right)-A\left(\eta_{2}\right)\right) Y\right)}_{(I I)} \\
& +\underbrace{M\left[\operatorname{tr}\left(A\left(\eta_{1}\right)\right)+\operatorname{tr}\left(A\left(\eta_{2}\right)\right)\right.}_{(I I I)}] .
\end{aligned}
$$


Estimate of (I). Remark that all the eigenvalues of $X-Y$ are non positive. Applying the previous matrix inequality (A.2) to the vector $(\xi,-\xi)$ where $\xi:=\frac{x_{1}-y_{1}}{\left|x_{1}-y_{1}\right|}$ and using (A.3), we obtain

$$
\langle(X-Y) \xi, \xi\rangle \leq 4\left\langle B^{\tau} \xi, \xi\right\rangle \leq 8 L \beta\left|x_{1}-y_{1}\right|^{\beta-2}\left(\frac{\beta-1}{3-\beta}\right)<0 .
$$

This means that at least one of the eigenvalue of $X-Y$ that we denote by $\lambda_{i_{0}}$ is negative and smaller than $8 L \beta\left|x_{1}-y_{1}\right|^{\beta-2}\left(\frac{\beta-1}{3-\beta}\right)$. The eigenvalues of $A\left(\eta_{1}\right)$ belong to $[\min (1, p-1), \max (1, p-1)]$. Using $($ A.7), it results that

$$
\begin{aligned}
\operatorname{tr}\left(A\left(\eta_{1}\right)(X-Y)\right) & \leq \sum_{i} \lambda_{i}\left(A\left(\eta_{1}\right)\right) \lambda_{i}(X-Y) \\
& \leq \min (1, p-1) \lambda_{i_{0}}(X-Y) \\
& \leq \min (1, p-1) 8 L \beta\left|x_{1}-y_{1}\right|^{\beta-2}\left(\frac{\beta-1}{3-\beta}\right) .
\end{aligned}
$$

Estimate of (II). We have

$$
\begin{aligned}
A\left(\eta_{1}\right)-A\left(\eta_{2}\right) & =\left(\bar{\eta}_{1} \otimes \bar{\eta}_{1}-\bar{\eta}_{2} \otimes \bar{\eta}_{2}\right)(p-2) \\
& =\left[\left(\bar{\eta}_{1}-\bar{\eta}_{2}\right) \otimes \bar{\eta}_{1}-\bar{\eta}_{2} \otimes\left(\bar{\eta}_{2}-\bar{\eta}_{1}\right)\right](p-2)
\end{aligned}
$$

and it follows that

$$
\begin{aligned}
\operatorname{tr}\left(\left(A\left(\eta_{1}\right)-A\left(\eta_{2}\right)\right) Y\right) & \leq n\|Y\| \| A\left(\eta_{1}\right)-A\left(\eta_{2}\right)|| \\
& \leq n|p-2|\|Y\|\left|\bar{\eta}_{1}-\bar{\eta}_{2}\right|\left(\left|\bar{\eta}_{1}\right|+\left|\bar{\eta}_{2}\right|\right) \\
& \leq 2 n|p-2|\|Y\|\left|\bar{\eta}_{1}-\bar{\eta}_{2}\right| .
\end{aligned}
$$

By using (A.5) and $\left|\eta_{1}-\eta_{2}\right| \leq 4 M$ (since $\left|x_{1}-2 x_{0}+y_{1}\right| \leq 4$ ), we have

$$
\begin{aligned}
\left|\bar{\eta}_{1}-\bar{\eta}_{2}\right| & =\left|\frac{\eta_{1}}{\left|\eta_{1}\right|}-\frac{\eta_{2}}{\left|\eta_{2}\right|}\right| \leq \max \left(\frac{\left|\eta_{2}-\eta_{1}\right|}{\left|\eta_{2}\right|}, \frac{\left|\eta_{2}-\eta_{1}\right|}{\left|\eta_{1}\right|}\right) \\
& \left.\leq \frac{16 M}{\beta L\left|x_{1}-y_{1}\right|}\right)
\end{aligned}
$$

Combining the previous estimate with (A.4), we obtain

$$
\operatorname{tr}\left(\left(A\left(\eta_{1}\right)-A\left(\eta_{2}\right)\right) Y\right) \leq 64 n|p-2| M\left|x_{1}-y_{1}\right|^{-1} .
$$

Estimate of (III). We have

$$
M\left(\operatorname{tr}\left(A\left(\eta_{1}\right)\right)+\operatorname{tr}\left(A\left(\eta_{2}\right)\right)\right) \leq 2 M n \max (1, p-1) .
$$

Gathering the previous estimates with (A.6), we get

$$
\begin{aligned}
0 & \leq 2 C(\beta)\left(L\left|x_{1}-y_{1}\right|^{\beta-1}\right)^{-\gamma}|| f \|_{L^{\infty}\left(B_{1}\right)}|q|^{-\gamma-1}+64 n|p-2| M\left|x_{1}-y_{1}\right|^{-1} \\
& +2 M n \max (1, p-1)+\min (1, p-1) 8 L \beta\left|x_{1}-y_{1}\right|^{\beta-2}\left(\frac{\beta-1}{3-\beta}\right) .
\end{aligned}
$$


Choosing $L$ satisfying

$L \geq C(p, n)\left(M+\|f\|_{L^{\infty}\left(B_{1}\right)}^{\frac{1}{1+\gamma}}|q|^{-1}\right) \geq C(p, n)\left(\|\tilde{w}\|_{L^{\infty}\left(B_{1}\right)}+\|f\|_{L^{\infty}\left(B_{1}\right)}^{\frac{1}{\gamma+1}}|q|^{-1}\right)$,

we get

$$
0 \leq \frac{\min (1, p-1) \beta(\beta-1)}{200(3-\beta)} L\left|x_{1}-y_{1}\right|^{\beta-2}<0
$$

which is a contradiction and hence $\Phi(x, y) \leq 0$ for $(x, y) \in B_{r} \times B_{r}$. The desired result follows since for $x_{0}, y_{0} \in B_{\frac{3}{4}}$, we have $\Phi\left(x_{0}, x_{0}\right) \leq 0$, so we get

$$
\left|\tilde{w}\left(x_{0}\right)-\tilde{w}\left(y_{0}\right)\right| \leq L\left|x_{0}-y_{0}\right|^{\beta} .
$$

We conclude that $\tilde{w}$ is Hölder continuous in $B_{\frac{3}{4}}$ and

$$
|\tilde{w}(x)-\tilde{w}(y)| \leq C(n, p, \beta, \gamma)\left(\|\tilde{w}\|_{L^{\infty}\left(B_{1}\right)}+\frac{\|f\|_{L^{\infty}\left(B_{1}\right)}^{\frac{1}{1+\gamma}}}{|q|}\right)|x-y|^{\beta} .
$$

Appendix B. Uniform estimates for the approximating Problem

Proposition B.1. Let $v_{\varepsilon}$ be a smooth solution of (4.12) with $\gamma \in[0, \infty)$, $p \in(1, \infty)$ and $\varepsilon \in(0,1)$. Then there exists a positive constants $\bar{\alpha}>0$ and $C_{\bar{\alpha}}=C_{\bar{\alpha}}\left(n, p, \gamma,\|h\|_{L^{\infty}\left(\Omega^{\prime}\right)},\left\|v_{\varepsilon}\right\|_{L^{\infty}\left(\Omega^{\prime}\right)}\right)$ such that for every $x, y \in \Omega^{\prime}$, we have

$$
\left|v_{\varepsilon}(x)-v_{\varepsilon}(y)\right| \leq C_{\bar{\alpha}}|x-y|^{\bar{\alpha}} .
$$

Proof. The uniform interior estimate follows from [29, Theorem 1.1], [24, Theorem 1.1] and [31, Corollary 2] since the operator

$$
F(x, s, \eta, X):=-\left(|\eta|^{2}+\varepsilon^{2}\right)^{\frac{\gamma}{2}}\left[\operatorname{tr}(X)-(p-2) \frac{X \eta \cdot \eta}{|\eta|^{2}+\varepsilon^{2}}\right]+\lambda s-h_{\varepsilon}
$$

satisfies

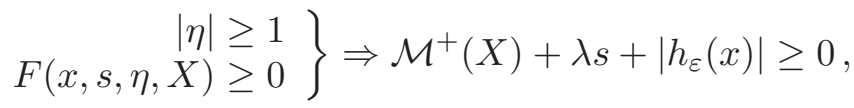

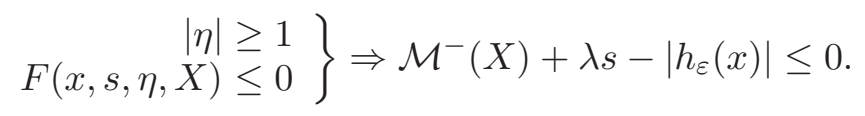

We are going to make use of the above Hölder estimate and the Ishii-Lions' method [20] again to prove the following Lipschitz estimate.

Proposition B.2. Let $v_{\varepsilon}$ be a smooth solution of (4.12) with $\gamma \in[0, \infty)$, $p \in(1, \infty)$ and $\varepsilon \in(0,1)$. Then there exists a positive constant $C=$ $C\left(n, p, \gamma,\left\|h_{\varepsilon}\right\|_{L^{\infty}\left(\Omega^{\prime}\right)},\left\|v_{\varepsilon}\right\|_{L^{\infty}\left(\Omega^{\prime}\right)}\right)$ such that for every $x, y \in \Omega^{\prime \prime}$, we have

$$
\left|v_{\varepsilon}(x)-v_{\varepsilon}(y)\right| \leq C|x-y| \text {. }
$$


Proof. Using a scaling and a translation argument combined with a covering argument, it is enough to prove the Lemma in the unit ball $B_{1}$ and for osc $v_{\varepsilon} \leq 1$ in $B_{1}$.

Like in the proof of the Hölder continuity (see Lemma 3.1), we fix $x_{0}, y_{0} \in$ $B_{3 / 4}$ and introduce the auxiliary function

$$
\Phi(x, y):=v_{\varepsilon}(x)-v_{\varepsilon}(y)-L \phi(|x-y|)-\frac{M}{2}\left|x-x_{0}\right|^{2}-\frac{M}{2}\left|y-y_{0}\right|^{2},
$$

where $\phi$ is defined below. Our goal is to show that $\Phi(x, y) \leq 0$ for $(x, y) \in$ $B_{r} \times B_{r}$, where $r=\frac{4}{5}$. We take

$$
\phi(t)= \begin{cases}t-t^{\delta} \phi_{0} & 0 \leq t \leq t_{1}:=\left(\frac{1}{\delta \phi_{0}}\right)^{1 /(\delta-1)} \\ \phi\left(t_{1}\right) & \text { otherwise }\end{cases}
$$

where $2>\delta>1$ and $\phi_{0}>0$ is such that $t_{1} \geq 2$ and $\gamma \phi_{0} 2^{\delta-1} \leq 1 / 4$.

Then

$$
\begin{aligned}
\phi^{\prime}(t) & = \begin{cases}1-\delta t^{\delta-1} \phi_{0} & 0 \leq t \leq t_{1} \\
0 & \text { otherwise }\end{cases} \\
\phi^{\prime \prime}(t) & = \begin{cases}-\delta(\delta-1) t^{\delta-2} \phi_{0} & 0<t \leq t_{1} \\
0 & \text { otherwise }\end{cases}
\end{aligned}
$$

In particular, $\phi^{\prime}(t) \in\left[\frac{3}{4}, 1\right]$ and $\phi^{\prime \prime}(t)<0$ when $t \in[0,2]$.

We argue by contradiction and assume that $\Phi$ has a positive maximum at some point $\left(x_{1}, y_{1}\right) \in \bar{B}_{r} \times \bar{B}_{r}$. Since $v_{\varepsilon}$ is continuous and its oscillation is bounded by 1 , we get

$$
\begin{gathered}
M\left|x_{1}-x_{0}\right|^{2} \leq 2 \operatorname{osc}_{B_{1}} v_{\varepsilon} \leq 2, \\
M\left|y_{1}-y_{0}\right|^{2} \leq 2 \operatorname{osc}_{B_{1}} v_{\varepsilon} \leq 2 .
\end{gathered}
$$

Notice that $x_{1} \neq y_{1}$. Choosing $M \geq\left(\frac{32}{r}\right)^{2}$, we have that $\left|x_{1}-x_{0}\right|<r / 16$ and $\left|y_{1}-y_{0}\right|<r / 16$ so that $x_{1}$ and $y_{1}$ are in $B_{r}$.

Using the fact that $v_{\varepsilon}$ is Hölder continuous, it follows, adjusting the constants (by choosing $2 M \leq C_{\bar{\alpha}}$ ), that

$$
\begin{gathered}
M\left|x_{1}-x_{0}\right| \leq C_{\bar{\alpha}}\left|x_{1}-y_{1}\right|^{\beta / 2}, \\
M\left|y_{1}-y_{0}\right| \leq C_{\bar{\alpha}}\left|x_{1}-y_{1}\right|^{\beta / 2} .
\end{gathered}
$$

The Jensen-Ishii's lemma ensures that

$$
\begin{aligned}
& \left(\zeta_{x}, X\right) \in \overline{\mathcal{J}}^{2,+}\left(v_{\varepsilon}\left(x_{1}\right)-\frac{M}{2}\left|x_{1}-x_{0}\right|^{2}\right), \\
& \left(\zeta_{y}, Y\right) \in \overline{\mathcal{J}}^{2,-}\left(v_{\varepsilon}\left(y_{1}\right)+\frac{M}{2}\left|y_{1}-y_{0}\right|^{2}\right),
\end{aligned}
$$


that is

$$
\begin{aligned}
& (a, X+M I) \in \overline{\mathcal{J}}^{2,+} v_{\varepsilon}\left(x_{1}\right), \\
& (b, Y-M I) \in \overline{\mathcal{J}}^{2,-} v_{\varepsilon}\left(y_{1}\right),
\end{aligned}
$$

where $\left(\zeta_{x}=\zeta_{y}\right)$

$$
\begin{gathered}
a=L \phi^{\prime}\left(\left|x_{1}-y_{1}\right|\right) \frac{x_{1}-y_{1}}{\left|x_{1}-y_{1}\right|}+M\left(x_{1}-x_{0}\right)=\zeta_{x}+M\left(x_{1}-x_{0}\right), \\
b=L \phi^{\prime}\left(\left|x_{1}-y_{1}\right|\right) \frac{x_{1}-y_{1}}{\left|x_{1}-y_{1}\right|}-M\left(y_{1}-y_{0}\right)=\zeta_{y}-M\left(y_{1}-y_{0}\right) .
\end{gathered}
$$

If $L$ is large enough (depending on the Hölder constant $C_{\bar{\alpha}}$ ), we have

$$
2 L \geq \sqrt{|a|^{2}+\varepsilon^{2}}, \sqrt{|b|^{2}+\varepsilon^{2}} \geq L \phi^{\prime}\left(\left|x_{1}-y_{1}\right|\right)-C_{\bar{\alpha}}\left|x_{1}-y_{1}\right|^{\bar{\alpha} / 2} \geq \frac{L}{2} .
$$

The Jensen-Ishii's lemma ensures that for any $\tau>0$, we can take $X, Y \in \mathcal{S}^{n}$ such that

$$
-[\tau+2\|B\|]\left(\begin{array}{cc}
I & 0 \\
0 & I
\end{array}\right) \leq\left(\begin{array}{cc}
X & 0 \\
0 & -Y
\end{array}\right)
$$

and

$$
\begin{aligned}
\left(\begin{array}{cc}
X & 0 \\
0 & -Y
\end{array}\right) & \leq\left(\begin{array}{cc}
B & -B \\
-B & B
\end{array}\right)+\frac{2}{\tau}\left(\begin{array}{cc}
B^{2} & -B^{2} \\
-B^{2} & B^{2}
\end{array}\right) \\
& =D^{2} \phi\left(x_{1}, y_{1}\right)+\frac{1}{\tau}\left(D^{2} \phi\left(x_{1}, y_{1}\right)\right)^{2}
\end{aligned}
$$

where

$$
\begin{aligned}
B=L \phi^{\prime \prime}\left(\left|x_{1}-y_{1}\right|\right) \frac{x_{1}-y_{1}}{\left|x_{1}-y_{1}\right|} \otimes \frac{x_{1}-y_{1}}{\left|x_{1}-y_{1}\right|} \\
+\frac{L \phi^{\prime}\left(\left|x_{1}-y_{1}\right|\right)}{\left|x_{1}-y_{1}\right|}\left(I-\frac{x_{1}-y_{1}}{\left|x_{1}-y_{1}\right|} \otimes \frac{x_{1}-y_{1}}{\left|x_{1}-y_{1}\right|}\right)
\end{aligned}
$$

and

$$
\begin{array}{r}
B^{2}=\frac{L^{2}\left(\phi^{\prime}\left(\left|x_{1}-y_{1}\right|\right)\right)^{2}}{\left|x_{1}-y_{1}\right|^{2}}\left(I-\frac{x_{1}-y_{1}}{\left|x_{1}-y_{1}\right|} \otimes \frac{x_{1}-y_{1}}{\left|x_{1}-y_{1}\right|}\right) \\
+L^{2}\left(\phi^{\prime \prime}\left(\left|x_{1}-y_{1}\right|\right)\right)^{2} \frac{x_{1}-y_{1}}{\left|x_{1}-y_{1}\right|} \otimes \frac{x_{1}-y_{1}}{\left|x_{1}-y_{1}\right|} .
\end{array}
$$

Observe that $\phi^{\prime \prime}(t)+\frac{\phi^{\prime}(t)}{t} \geq 0, \phi^{\prime \prime}(t) \leq 0$ for $t \in(0,2)$ and hence

$$
\begin{gathered}
\|B\| \leq L \phi^{\prime}\left(\left|x_{1}-y_{1}\right|\right), \\
\left\|B^{2}\right\| \leq L^{2}\left(\left|\phi^{\prime \prime}\left(\left|x_{1}-y_{1}\right|\right)\right|+\frac{\phi^{\prime}\left(\left|x_{1}-y_{1}\right|\right)}{\left|x_{1}-y_{1}\right|}\right)^{2} .
\end{gathered}
$$

Besides, for $\xi=\frac{x_{1}-y_{1}}{\left|x_{1}-y_{1}\right|}$, we have

$$
\langle B \xi, \xi\rangle=L \phi^{\prime \prime}\left(\left|x_{1}-y_{1}\right|\right)<0, \quad\left\langle B^{2} \xi, \xi\right\rangle=L^{2}\left(\phi^{\prime \prime}\left(\left|x_{1}-y_{1}\right|\right)\right)^{2} .
$$


Taking $\tau=4 L\left(\left|\phi^{\prime \prime}\left(\left|x_{1}-y_{1}\right|\right)\right|+\frac{\phi^{\prime}\left(\left|x_{1}-y_{1}\right|\right)}{\left|x_{1}-y_{1}\right|}\right)$, we obtain for $\xi=\frac{x_{1}-y_{1}}{\left|x_{1}-y_{1}\right|}$,

$$
\begin{aligned}
\langle B \xi, \xi\rangle+\frac{2}{\tau}\left\langle B^{2} \xi, \xi\right\rangle & =L\left(\phi^{\prime \prime}\left(\left|x_{1}-y_{1}\right|\right)+\frac{2}{\tau} L\left(\phi^{\prime \prime}\left(\left|x_{1}-y_{1}\right|\right)\right)^{2}\right) \\
& \leq \frac{L}{2} \phi^{\prime \prime}\left(\left|x_{1}-y_{1}\right|\right)<0
\end{aligned}
$$

Applying inequalities (B.8) and (B.9) to any vector $(\xi, \xi)$ with $|\xi|=1$, we have that $X-Y \leq 0$ and $\|X\|,\|Y\| \leq 2\|B\|+\tau$.

We denote $\eta_{1}=\sqrt{|a|^{2}+\varepsilon^{2}}, \eta_{2}=\sqrt{|b|^{2}+\varepsilon^{2}}$ and we write the viscosity inequalities

$$
\begin{gathered}
-f\left(x_{1}\right)\left|\eta_{1}\right|^{-\gamma} \leq \operatorname{tr}(X+M I)+(p-2) \frac{\langle(X+M I) a, a\rangle}{\left|\eta_{1}\right|^{2}} \\
f\left(y_{1}\right)\left|\eta_{2}\right|^{-\gamma} \geq \operatorname{tr}(Y-M I)+(p-2) \frac{\langle(Y-M I) b, b\rangle}{\left|\eta_{2}\right|^{2}} .
\end{gathered}
$$

We end up with

$$
\begin{gathered}
-f\left(x_{1}\right)\left|\eta_{1}\right|^{-\gamma} \leq \operatorname{tr}(D(a)(X+M I)) \\
f\left(y_{1}\right)\left|\eta_{2}\right|^{-\gamma} \leq-\operatorname{tr}(D(b)(Y-M I))
\end{gathered}
$$

where

$$
D(\eta):=I+(p-2) \frac{\eta \otimes \eta}{|\eta|^{2}+\varepsilon^{2}} .
$$

Adding the two inequalities and using that $\left|\eta_{1}\right|^{-\delta},\left|\eta_{2}\right|^{-\delta} \leq c L^{-\delta}$, we get

$$
-2 c\|f\|_{L^{\infty}\left(B_{1}\right)} L^{-\delta} \leq \operatorname{tr}(D(a)(X+M I))-\operatorname{tr}(D(b)(Y-M I)) .
$$

It follows that

$$
\begin{aligned}
-2 c\|f\|_{L^{\infty}\left(B_{1}\right)} L^{-\gamma} \leq & \underbrace{\operatorname{tr}(D(a)(X-Y))}_{(I)}+\underbrace{\operatorname{tr}((D(a)-D(b)) Y)}_{(I I)} \\
& +\underbrace{M[\operatorname{tr}(D(a))+\operatorname{tr}(D(b))] .}_{(I I I)}
\end{aligned}
$$

Estimate of (I). Observe that all the eigenvalues of $X-Y$ are non positive. Moreover, applying the previous matrix inequality (B.9) to the vector $(\xi,-\xi)$ where $\xi:=\frac{x_{1}-y_{1}}{\left|x_{1}-y_{1}\right|}$ and using (B.12), we obtain

$$
\begin{aligned}
\langle(X-Y) \xi, \xi\rangle & \left.\leq 4\left(\langle B \xi, \xi\rangle+\frac{2}{\tau}\left\langle B^{2} \xi, \xi\right\rangle\right)\right) \\
& \leq 2 L \phi^{\prime \prime}\left(\left|x_{1}-y_{1}\right|\right)<0 .
\end{aligned}
$$

We deduce that at least one of the eigenvalue of $X-Y$ denoted by $\lambda_{i_{0}}$ is negative and smaller than $2 L \phi^{\prime \prime}\left(\left|x_{1}-y_{1}\right|\right)$. The eigenvalues of $D(a)$ belong 
to $[\min (1, p-1), \max (1, p-1)]$. Using (B.16), it follows that

$$
\begin{aligned}
\operatorname{tr}(D(a)(X-Y)) & \leq \sum_{i} \lambda_{i}(D(a)) \lambda_{i}(X-Y) \\
& \leq \min (1, p-1) \lambda_{i_{0}}(X-Y) \\
& \leq 2 \min (1, p-1) L \phi^{\prime \prime}\left(\left|x_{1}-y_{1}\right|\right) .
\end{aligned}
$$

Estimate of (II). We have

$$
\begin{aligned}
D(a)-D(b) & =\left(\frac{a}{\eta_{1}} \otimes \frac{a}{\eta_{1}}-\frac{b}{\eta_{2}} \otimes \frac{b}{\eta_{2}}\right)(p-2) \\
& =\left[\left(\frac{a}{\eta_{1}}-\frac{b}{\eta_{2}}\right) \otimes \frac{a}{\eta_{1}}+\frac{b}{\eta_{2}} \otimes\left(\frac{a}{\eta_{1}}-\frac{b}{\eta_{2}}\right)\right](p-2) .
\end{aligned}
$$

It follows that

$$
|D(a)-D(b)| \leq 2|p-2|\left|\frac{a}{\eta_{1}}-\frac{b}{\eta_{2}}\right|
$$

Hence,

$$
\begin{aligned}
\operatorname{tr}((D(a)-D(b)) Y) & \leq n\|Y\|\|D(a)-D(b)\| \\
& \leq 2 n|p-2|\|Y\|\left|\frac{a}{\eta_{1}}-\frac{b}{\eta_{2}}\right|
\end{aligned}
$$

We have

$$
\begin{aligned}
\left|\frac{a}{\eta_{1}}-\frac{b}{\eta_{2}}\right| & \leq 2 \max \left(\frac{|a-b|}{\left|\eta_{2}\right|}, \frac{|a-b|}{\left|\eta_{1}\right|}\right) \\
& \leq \frac{8 C_{\bar{\alpha}}}{L}\left|x_{1}-y_{1}\right|^{\bar{\alpha} / 2},
\end{aligned}
$$

where we used (B.7) and (B.6).

Using the estimates (B.8)-(B.11), we obtain

$$
\begin{aligned}
\|Y\| & =\max _{\bar{\xi}}|\langle Y \bar{\xi}, \bar{\xi}\rangle| \leq 2|\langle B \bar{\xi}, \bar{\xi}\rangle|+\frac{4}{\tau}\left|\left\langle B^{2} \bar{\xi}, \bar{\xi}\right\rangle\right| \\
& \leq 4 L\left(\frac{\phi^{\prime}\left(\left|x_{1}-y_{1}\right|\right)}{\left|x_{1}-y_{1}\right|}+\left|\phi^{\prime \prime}\left(\left|x_{1}-y_{1}\right|\right)\right|\right) .
\end{aligned}
$$

Hence, remembering that $\left|x_{1}-y_{1}\right| \leq 2$, we end up with

$$
\begin{aligned}
\operatorname{tr}((D(a)-D(b)) Y) \leq & 128 n|p-2| C_{\bar{\alpha}} \phi^{\prime}\left(\left|x_{1}-y_{1}\right|\right)\left|x_{1}-y_{1}\right|^{-1+\bar{\alpha} / 2} \\
& +128 n|p-2| C_{\bar{\alpha}}\left|\phi^{\prime \prime}\left(\left|x_{1}-y_{1}\right|\right)\right| .
\end{aligned}
$$

Estimate of (III). Finally, we have

$$
M(\operatorname{tr}(D(a))+\operatorname{tr}(D(b))) \leq 2 M n \max (1, p-1) .
$$


Putting together the previous estimates with (B.15) and recalling the definition of $\phi$, we end up with

$$
\begin{aligned}
0 \leq & 128 n|p-2| C_{\bar{\alpha}}\left(\phi^{\prime}\left(\left|x_{1}-y_{1}\right|\right)\left|x_{1}-y_{1}\right|^{\bar{\alpha} / 2-1}+\left|\phi^{\prime \prime}\left(\left|x_{1}-y_{1}\right|\right)\right|\right) \\
& +2 \min (1, p-1) L \phi^{\prime \prime}\left(\left|x_{1}-y_{1}\right|\right)++2 M n \max (1, p-1)-2 c L^{-\gamma}\|f\|_{L^{\infty}\left(B_{1}\right)} \\
\leq & 128 n|p-2| C_{\bar{\alpha}}\left|x_{1}-y_{1}\right|^{\bar{\alpha} / 2-1}+2 n M \max (1, p-1) \\
& +128 n|p-2| C_{\bar{\alpha}} \delta(\delta-1) \phi_{0}\left|x_{1}-y_{1}\right|^{\delta-2}-2 c L^{-\gamma}\|f\|_{L^{\infty}\left(B_{1}\right)} \\
& -2 \min (1, p-1) \delta(\delta-1) \phi_{0} L\left|x_{1}-y_{1}\right|^{\delta-2} .
\end{aligned}
$$

Taking $\delta=1+\bar{\alpha} / 2>1$ and choosing $L$ large enough depending on $p, n, C_{\beta}$, $\|f\|_{L^{\infty}}$ we get

$$
0 \leq \frac{-\min (1, p-1) \delta(\delta-1) \phi_{0}}{200} L\left|x_{1}-y_{1}\right|^{\delta-2}-2 c L^{-\gamma}\|f\|_{L^{\infty}\left(B_{1}\right)}<0,
$$

which is a contradiction. Hence $\Phi(x, y) \leq 0$ for $(x, y) \in B_{r} \times B_{r}$. The desired result follows since for $x_{0}, y_{0} \in B_{\frac{3}{4}}$, we have $\Phi\left(x_{0}, y_{0}\right) \leq 0$, we get

$$
\left|v_{\varepsilon}\left(x_{0}\right)-v_{\varepsilon}\left(y_{0}\right)\right| \leq L \phi\left(\left|x_{0}-y_{0}\right|\right) \leq L\left|x_{0}-y_{0}\right| .
$$

Proposition B.3. Let $v_{\varepsilon}$ be a smooth solution of (4.12) with $\gamma \in(-1,0]$, $p \in(1, \infty)$ and $\varepsilon \in(0,1)$. Then there exists a positive constant $C=$ $C\left(n, p, \gamma,\left\|h_{\varepsilon}\right\|_{L^{\infty}\left(\Omega^{\prime}\right)},\left\|v_{\varepsilon}\right\|_{L^{\infty}\left(\Omega^{\prime}\right)}\right)$ such that for every $x, y \in \Omega^{\prime \prime}$, we have

$$
\left|v_{\varepsilon}(x)-v_{\varepsilon}(y)\right| \leq C|x-y| \text {. }
$$

Proof. Recall that $v_{\varepsilon}$ are Hölder continuous solutions of (4.7) with uniform Hölder bound

$$
C(p, n, \gamma)\left(\left\|v_{\varepsilon}\right\|_{L^{\infty}\left(\Omega^{\prime}\right)}+\left\|\bar{h}_{\varepsilon}\right\|_{L^{\infty}\left(\Omega^{\prime}\right)}+\|f\|_{L^{\infty}\left(\Omega^{\prime}\right)}^{\frac{-1}{1+\gamma}}\right) .
$$

The proof proceeds similarly to the previous proof of Proposition B.2. The main changes occur when writing the viscosity inequalities: Now we write

$$
\begin{aligned}
-\bar{h}_{\varepsilon}\left(x_{1}\right)-f\left(x_{1}\right)\left|\eta_{1}\right|^{-\gamma} & \leq \operatorname{tr}(D(a)(X+M I)) \\
\bar{h}_{\varepsilon}\left(y_{1}\right)+f\left(y_{1}\right)\left|\eta_{2}\right|^{-\gamma} & \leq-\operatorname{tr}(D(b)(Y-M I)) .
\end{aligned}
$$

and adding the two inequalities we have

$$
-2\left\|\bar{h}_{\varepsilon}\right\|_{L^{\infty}\left(\Omega^{\prime}\right)}-2 c\|f\|_{L^{\infty}\left(\Omega^{\prime}\right)} L^{-\delta} \leq \operatorname{tr}(D(a)(X+M I))-\operatorname{tr}(D(b)(Y-M I)) .
$$

By choosing

$$
L \geq c\left(\left\|v_{\varepsilon}\right\|_{L^{\infty}\left(\Omega^{\prime}\right)}+\left\|\bar{h}_{\varepsilon}\right\|_{L^{\infty}\left(\Omega^{\prime}\right)}+\|f\|_{L^{\infty}\left(\Omega^{\prime}\right)}^{\frac{-1}{1+\gamma}}\right),
$$

the proof is completed as the proof of Proposition B.2. 


\section{REFERENCES}

[1] E. Acerbi, N. Fusco, Regularity for minimizers of non-quadratic functionals: the case $1<p<2$. J. Math. Anal. Appl., 140, 115-135, 1989.

[2] A. Attouchi, M. Parviainen, E. Ruosteenoja, $C^{1, \alpha}$ regularity for the normalized p-Poisson problem. J. Math. Pures. Appl. 108(9):553-591, 2017.

[3] L. Bers, L. Nirenberg, On linear and non-linear elliptic boundary value problems in the plane. Convegno Internazionale sulle Equazioni Lineari alle Derivate Parziali, Trieste, 1954, pp. 141-167, Edizioni Cremonese, Roma, 1955.

[4] H. Beirão DA Veiga, F. Crispo, On the global $W^{2, q}$ regularity for nonlinear $N$-systems of the $p$-Laplacian type in $n$ space variables. Nonlinear Anal. $75(11)$ : 4346-4354, 2012.

[5] H. Beirão dA VEIgA, F. CRispo, On the global regularity for nonlinear systems of the $p$-Laplacian type. Discrete Contin. Dyn. Syst. Ser. S 6 (5):1173-1191, 2013.

[6] I. Birindelli, F. Demengel, Comparison principle and Liouville type results for singular fully nonlinear operators. Ann. Fac. Sci Toulouse Math, (6) 13 no.2, 261287, 2004.

[7] I. Birindelli, F. Demengel, First Eigenvalue and Maximum Principle for fully nonlinear singular operators. Advances in Partial Diff. equations 11 (1):91-119, 2006.

[8] I. Birindelli, F. Demengel, The Dirichlet problem for singular fully nonlinear operators. Discrete and Continuous Dynamical Systems. Supplement, 110-121, 2007.

[9] I. Birindelli, F. Demengel, Regularity and uniqueness of the first eigenfunction for singular fully nonlinear operators. J. Differential Equations, 249(5):1089-1110, 2010.

[10] I. Birindelli, F. Demengel, Regularity for radial solutions of degenerate fully nonlinear equations, Nonlinear Anal, 75(17):6237-6249, 2012.

[11] I. Birindelli, F. Demengel, $C^{1, \beta}$ regularity for Dirichlet problems associated to fully nonlinear degenerate elliptic equations. ESAIM Control Optim. Calc. Var. 20(4): 1009-1024, 2014.

[12] B. BojArski, T. Iwaniec, $p$-harmonic equation and quasiregular mappings. Partial differential equations (Warsaw, 1984), Banach Center Publ., 19, PWN, Warsaw 2538, 1987.

[13] L.A. CAffarelli, X. CABrÉ, Fully nonlinear elliptic equations. volume 43 of American Mathematical Society Colloquium Publications. American Mathematical Society, Providence, RI, 1995.

[14] S. Campanato, Un risultato relativo ad equazioni ellittiche del secondo ordine di tipo non variazionale. (Italian) Ann. Scuola Norm. Sup. Pisa (3) 21: 701-707, 1967.

[15] A. Cellina, The regularity of solutions to some variational problems, including the $p$-Laplace equation for $2 \leq p<3$. To appear in ESAIM: Control. Optim. Calc. Var.

[16] Y. G. Chen, Y. Giga, S. Goto, Uniqueness and existence of viscosity solutions of generalized mean curvature flow equations. J. Differential Geom. 33: (3), 749-786, 1991.

[17] M.CHIcco, Dirichlet problem for a class of linear second order elliptic partial differential equations with discontinuous coefficients. Ann. Mat. Pura Appl. (4) 92: 13-22, 1972.

[18] H. O. Condes, Zero order a priori estimates for solutions of elliptic differential equations. Proc. Sympos. Pure Math. 4: 157-166, 1961.

[19] M. G. Crandall, Viscosity solutions: a primer. Viscosity solutions and applications (Montecatini Terme, 1995), Lecture Notes in Math., 1660, Springer, Berlin,1-43, 1997.

[20] M.G. Crandall, H. Ishit, P-L Lions, User's guide to viscosity solutions of second order partial differential equations. Bull. Am. Math. Soc., 27(1):1-67, 1992. 
[21] F. Crispo, P. Maremonti, Higher regularity of solutions to the $p$-Laplacian system in the subquadratic case. Riv. Math. Univ. Parma 5(1):39-63, 2014.

[22] L. Damascelli, B. Sciunzi, Regularity, monotonicity and symmetry of positive solutions of $m$-Laplace equations. J. Differential Equations, 206(2: 483--515, 2004.

[23] G. DÁvila, P. Felmer, A. QuaAs, Harnack inequality for singular fully nonlinear operators and some existence results. Calc. Var. Partial Differential Equations 39 , no. 3-4, 557-578, 2010.

[24] F. Delarue, Krylov and Safonov estimates for degenerate quasilinear elliptic PDEs. J. Differential Equations, 248(4):924-951, 2010.

[25] F. Duzane, G. Mingione, Local Lipschitz regularity for degenerate elliptic systems. Ann. Inst. H. Poincaré Anal. Non Linéaire, 27(6):1361-1396, 2010.

[26] L.C. Evans, J. Spruck, Motion of level sets by mean curvature. J. Differ. Geom. 33, 635-681, 1991.

[27] D. Gilbarg, N.S. Trudinger, Elliptic partial differential equations of second order. Classics in Mathematics. Springer-Verlag, Berlin, 2001.

[28] G. Gripenberg, Boundary regularity for viscosity solutions to degenerate elliptic PDEs. J. Math. Anal. Appl. 352(1): 175-183, 2009.

[29] C. Imbert, Alexandroff-Bakelman-Pucci estimate and Harnack inequality for degenerate/singular fully nonlinear elliptic equations. J. Differential Equations 250 (3): 1553-574, 2009.

[30] C. Imbert, L. Silvestre, $C^{1, \alpha}$ regularity of solutions of some degenerate fully nonlinear elliptic equations. Adv. Math., 233(1):196 - 206, 2013.

[31] C. Imbert, L. Silvestre, Estimates on elliptic equations that hold only where the gradient is large. J. Eur. Math. Soc. (JEMS) 18(6):1321-1338, 2016.

[32] H. Ishis, P-L Lions, Viscosity solutions of fully nonlinear second-order elliptic partial differential equations. J. Differential equations, 83(1):26-78, 1990.

[33] T. Iwaniec, J. J. Manfredi, Regularity of $p$-harmonic functions on the plane. Rev. Mat. Iberoamericana 5:1-19, 1989.

[34] V. Julin, P. Juutinen, A new proof for the equivalence of weak and viscosity solutions for the $p$-Laplace equation. Comm. Partial Differential Equations, 37(5):934946, 2012.

[35] P. Juntinen, P. Lindqvist, J. J. Manfredi, On the equivalence of viscosity solutions and weak solutions for a quasi-linear equation. SIAM J. Math. Anal., 33(3):699$717,2001$.

[36] B. Kawohl, J. J. Manfredi, M. Parviainen, Solutions of nonlinear PDEs in the sense of averages. J. Math. Pures. Appl., 97(2):173-188, 2012.

[37] O.A. Ladyzhenskaya, N.N. Uraltseva, Linear and quasilinear elliptic equations. Translated from the Russian by Scripta Technica, Inc. Translation editor: Leon Ehrenpreis. Academic Press, New York-London, 1968.

[38] T. Leonori, A. Porretta, G. Riey, Comparison principles for p-Laplace equations with lower order terms. Ann. Mat. Pura Appl. (4) 196 no. 3, 877-903, 2017.

[39] D. LI, Z. LI, Global $W^{2, \delta}$ estimates for a type of singular fully nonlinear elliptic equations. Mathematische Zeitschrift, 285.3-4:1167-1179, 2017.

[40] D. LI, Z. LI, Global $W^{2, \delta}$ estimates for singular fully nonlinear elliptic equations with $L^{n}$ right hand side terms. ArXiv preprint, 2017. https://arxiv.org/pdf/1709.04707.pdf

[41] G. M. Lieberman, Boundary regularity for solutions of degenerate elliptic equations. Nonlinear Anal. 12 (11): 1203-1219, 1988.

[42] F. H. Lin, Second derivative $L^{p}$-estimates for elliptic equations of nondivergent type. Proc. Amer. Math. Soc. 96 (3): 447-451, 1986.

[43] H. Lou, On singular sets of local solutions to p-Laplace equations. Chinese Ann. Math. Ser. B, 29(5) : 521-530, 2008.

[44] F. MA, L. WANG, Boundary first order derivative estimates for fully nonlinear elliptic equations. J. Differential Equations 252 (2): 988-1002, 2012. 
[45] J.J. Manfredi, A. Weitsman, On the Fatou theorem for p-harmonic function. Comm. Partial Differential Equations, 13(6): 651-668, 1988.

[46] C. Mercuri, G. Riey, B. Sciunzi, A regularity result for the $p$-Laplacian near uniform ellipticity. SIAM J. Math. Anal., 48(3): 2059-2075, 2016.

[47] A. Maugeri, D. K. Palagachev, G. L. Softova, Elliptic and parabolic equations with discontinuous coefficients. Mathematical Research, 109. Wiley-VCH Verlag Berlin GmbH, Berlin, 2000. 256 pp.

[48] M. Medina, P. OchoA, On viscosity and weak solutions for non-homogeneous pLaplace equations. Advances in Nonlinear Analysis, to appear 2017.

[49] P. Pucci, R. Servadei, Regularity of weak solutions of homogeneous or inhomogeneous quasilinear elliptic equations. Indiana Univ. Math. J. 57(7):3329-3363, 2008.

[50] B. ScIUnzI, Regularity and comparison principles for p-Laplace equations with vanishing source term. Commun. Contemp. Math., 16(6): 1450013, 20 pp, 2014.

[51] B. ScIunzI, Some results on the qualitative properties of positive solutions of quasilinear elliptic equations. NoDEA Nonlinear Differential Equations Appl. 14(3-4): 315$-334,2007$.

[52] J. Serrin, H. Zou, Cauchy-Liouville and universal boundedness theorems for quasilinear elliptic equations and inequalities. Acta Math. 189 (1): 79-142, 2002.

[53] G. Talenti, Sopra una classe di equazioni ellittiche a coefficienti misurabili, Ann. Mat. Pura Appl. (4) 69: 285-304, 1965.

[54] P. TOlksdorf, Regularity for a more general class of quasilinear elliptic equations. J. Differential Equations, 51(1):126-150, 1984.

[55] K. Uhlenbeck, Regularity for a class of non-linear elliptic systems. Acta Math., 138(1):219-240, 1977.

Department of Mathematics and Statistics, University of Jyväskylä, PO Box 35, FI-40014 JYVÄSKYLÄ, FinLAND

E-mail address: amalattouchi@gmail.com

Department of Mathematics and Statistics, University of Jyväskylä, PO Box 35, FI-40014 JYVÄSKYLÄ, FINLAND

E-mail address: eero.ruosteenoja@jyu.fi 